\title{
On colored Turaev-Viro invariants for links in arbitrary 3-manifolds
}

\author{
Ekaterina Pervova* $\quad$ Carlo Petronio**
}

November 19, 2018

\begin{abstract}
We consider certain invariants of links in 3-manifolds, obtained by a specialization of the Turaev-Viro invariants of 3-manifolds, that we call colored Turaev-Viro invariants. Their construction is based on a presentation of a pair $(M, L)$, where $M$ is a closed oriented 3-manifold and $L \subset M$ is an oriented link, by a triangulation of $M$ such that each component of $L$ is an edge. We analyze some basic properties of these invariants, including the behavior under connected sums of pairs away and along links. These properties allow us to provide examples of links in $\mathbb{S}^{3}$ having the same HOMFLY polynomial and the same Kauffman polynomial but distinct Turaev-Viro invariants, and similar examples for the Alexander polynomial. We also investigate the relations between the Turaev-Viro invariants of $(M, L)$ and those of $M \backslash L$, showing that they are sometimes but not always determined by each other.
\end{abstract}

MSC (2000): 57M27

\section{Introduction}

The ideas of quantum topology [18] have been repeatedly applied over the time to construct invariants of links in 3-manifolds. These constructions have

*The first author was supported by the Marie Curie International Incoming Fellowship MIIF1-CT-2006-038734 within the 6th European Community Framework Programme

${ }^{* *}$ The second author gratefully thanks the University of Melbourne, the Université Paul Sabatier in Toulouse, the Université Louis Pasteur in Strasbourg and the Columbia University in New York City, where part of this research was carried out 
led, among other things, to a computation of the Jones polynomial of links in $\mathbb{S}^{3}$ via a 2-dimensional [7, 16] or a 3-dimensional [17] state-sum model. In particular, in the latter model the invariants are calculated as certain statesums on triangulations of link exteriors, with state-sums based on the socalled quantum $6 j$-symbols associated with the quantum group $U_{q}\left(\operatorname{sl}_{2}(\mathbb{C})\right)[2$, 7]. The model furthermore takes into account a certain geometric picture on the boundary of the exterior.

A different approach was used in [3], based on the notion of a triangulation of a pair $(M, L)$, namely a triangulation of $M$ such that $L$ consists of edges, where $M$ is a 3 -manifold and $L$ is a link in $M$. The invariants are defined as state-sums on such triangulations, using the quantum $6 j$-symbols associated with $U_{q}\left(s l_{2}(\mathbb{C})\right)$ and restricting only to states such that the edges contained in $L$ have a certain pre-determined color. The main achievement of [3] with respect to these invariants was to establish a formula relating their Fourier transforms to the so-called relativistic spin network invariant, and to exhibit a relation with the Witten-Reshetikhin-Turaev invariant of a certain framed link in a 3-manifold (where the underlying link is different from the initial one).

The aim of the present paper is to study in greater detail the construction of [3], settling some of the issues not addressed there. To this end we re-define the invariants (that we call colored Turaev-Viro invariants) on the basis of algebraic initial data satisfying certain axioms (closely following the style of [19]). We note that our definition of initial data differs from that in [19] because of some further requirements that we introduce. We then investigate the behaviour of the invariants under the operations of connected sum of pairs away and along links (Theorems 4.8 and 4.9). This point was not touched in [3] even for the specific invariants considered there.

We then proceed to consider two instances of initial data, introduced in [19] and in [9, 10] respectively, that satisfy our additional requirements. The aim here is to test the effectiveness of the invariants by comparing them with other known ones. In particular, our computations show that for links in $\mathbb{S}^{3}$ the colored Turaev-Viro invariants are independent of many known ones, such as the HOMFLY polynomial, the Kauffman polynomial, and the Alexander polynomial. Indeed, for each of these polynomials we provide an example of pairs of links that are not distinguished by the polynomial but are distinguished by one of the colored Turaev-Viro invariants. Finally, we discuss relations with the invariants of the link complements, showing that they are sometimes but not always determined by each other. 
On technical grounds, we note that we restrict to triangulations such that each link component consists of one edge only. Furthermore, for the whole paper we employ the dual viewpoint of the so-called special spines [10]. More precisely, we present pairs $(M, L)$, where $M$ is a closed oriented 3-manifold and $L \subset M$ is an oriented link, by certain o-spines, which are oriented special skeleta of $M$ with some marked regions.

\section{Links, o-spines, and Turaev-Viro invariants of 3-manifolds}

In this section we recall some known definitions and facts that will be used in the rest of the paper.

Links in 3-manifolds In this paper we consider pairs $(M, L)$, where $M$ is an oriented closed 3-manifold and $L$ is an oriented link in $M$. For such pairs we consider the notions of ordinary connected sum and of link-connected sum.

We say that $(M, L)$ is the ordinary connected sum of $\left(M_{1}, L_{1}\right)$ and $\left(M_{2}, L_{2}\right)$ if it is homeomorphic to the pair obtained by removing from each $M_{i}$ a small open ball $B_{i}$ disjoint from $L_{i}$ and by identifying $\partial B_{1}$ with $\partial B_{2}$ via an orientation-reversing homeomorphism. We say that $(M, L)$ is the linkconnected sum of $\left(M_{1}, L_{1}\right)$ and $\left(M_{2}, L_{2}\right)$ if it is homeomorphic to the pair obtained by removing from $M_{i}$ a small open ball $B_{i}$ such that $B_{i} \cap L_{i}$ consists of one simple unknotted arc and identifying $\partial B_{1}$ with $\partial B_{2}$ via an orientationreversing homeomorphism such that $\partial B_{1} \cap L_{1}$ is identified with $\partial B_{2} \cap L_{2}$ in the only way that induces a consistent orientation on $L$.

Remark 1.1. For connected $M_{1}$ and $M_{2}$ the ordinary connected sum is welldefined and the link-connected sum depends only on the link components involved, so it is well-defined for knots.

O-spines The notion of o-spine extends the notion of a special spine of a 3 -manifold introduced in [5].

Definition 1.2. A polyhedron $P$ is called special if

- each point of $P$ has neighbourhood of type I, II, or III as shown in Fig. 1: 
- the connected components of the set of type I points are open discs (henceforth called regions);

- the connected components of the set of type II points are open intervals (henceforth called edges).
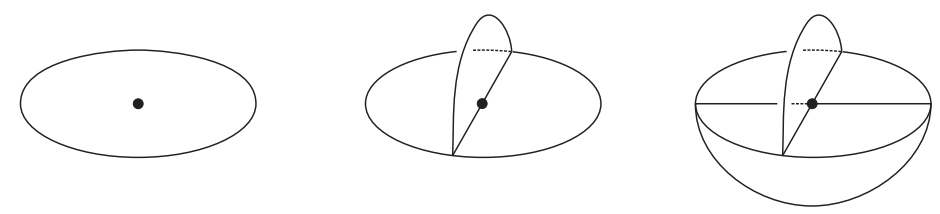

Figure 1: Type I, II, and III neighborhoods of points in a special polyhedron

Type III points are called vertices. The union of the edges and the vertices is called the singular graph of $P$ and is denoted by $S(P)$. We also denote the set of all vertices by $V(P)$, the set of all edges by $E(P)$, and the set of all regions by $R(P)$.

A subpolyhedron of a closed 3-manifold $M$ is called a spine of $M$ if its complement is homeomorphic to an open 3-ball. Two manifolds with homeomorphic spines need not be homeomorphic but they are if the spines are special [5]. For this and more results on special spines see [10].

An orientation on a special polyhedron, as defined in [4], is a choice of a "screw-orientation" along the edges (as in Fig. 2-left), with compatibility at vertices as in Fig. 2-right. If $P$ is a special spine of an orientable $M$, an orientation on $M$ determines one on $P$, and, conversely, an orientation on $P$ uniquely determines an orientation on $M$.

We define a marking on a special polyhedron $P$ to be the selection of some regions of $P$ together with the choice of an orientation for each selected region.
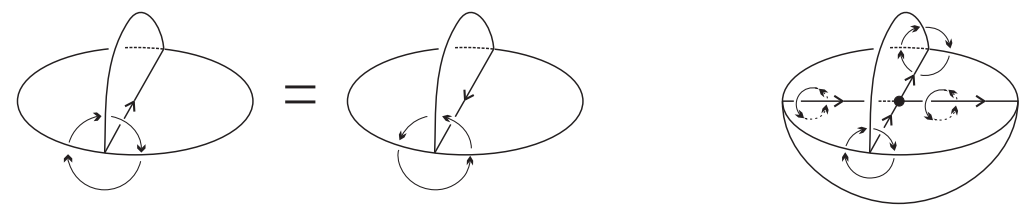

Figure 2: Convention on screw-orientations and compatibility at vertices for an oriented special polyhedron 

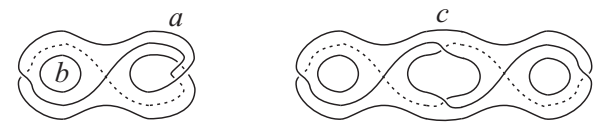

Figure 3: The abalone and Bing's House

Definition 1.3. If $M$ is a closed oriented 3-manifold and $L \subset M$ is an oriented link then a marked oriented special subpolyhedron $P$ of $M$ is called an o-spine of $(M, L)$ if:

1. $P$ and $M$ are consistently oriented;

2. $L$ intersects $P$ away from $S(P)$ and transversely;

3. Each connected component $B$ of $M \backslash P$ is a 3-ball and $B \cap L$ is either empty or an unknotted proper arc in $B$;

4. Each component of $L$ intersects $P$ at one point only;

5. The marked regions of $P$ are precisely those intersected by $L$, and they are oriented consistently with the orientations of $L$ and $M$.

Since each component of $L$ intersects only one (marked) region of $P$, we will call this region dual to the component.

We will always represent a special polyhedron $P$ by a picture suggesting how a regular neighbourhood of $S(P)$ is embedded in $\mathbb{S}^{3}$, and we will endow $P$ with the orientation induced by this embedding. Two examples are shown in Fig. 3. In both cases it is not hard to check that $P$ is a special spine of $\mathbb{S}^{3}$. The spine in Fig. 3-left is called the abalone and it has two regions $a$ and $b$. If we select the region $a$ and we give it any orientation, the resulting marked spine is an o-spine of the unknot, whereas if we select $b$ we get the right-handed trefoil (again the orientation of $b$ is immaterial). The spine in Fig. 3-right is called the Bing house. It has three regions, marking any of which we get an o-spine of the unknot. The following fact is known [1, 12].

Proposition 1.4. Every pair $(M, L)$ as above admits an o-spine.

Moves on o-spines Consider the moves MP and Lune shown in Fig. 4. Of course if we apply MP (which stands for "Matveev-Piergallini," see [10] for the appropriate references), or its inverse, to a special spine of a 3-manifold we 


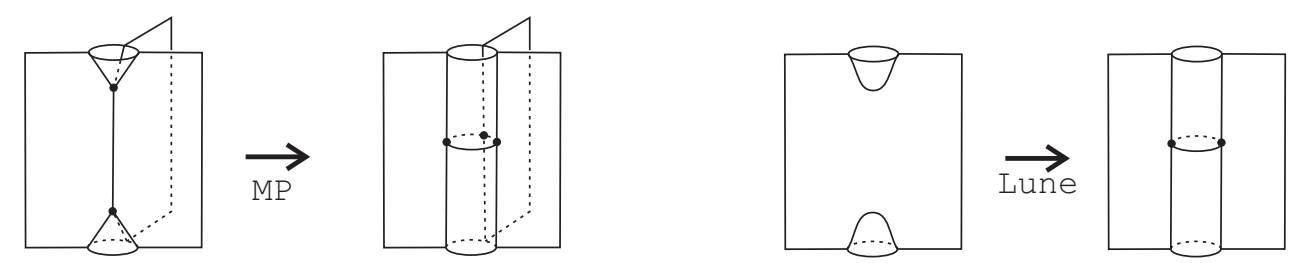

Figure 4: Moves on oriented special polyhedra, with local orientations induced by the embedding in $\mathbb{S}^{3}$

get a new special spine of the same manifold. Moreover, it is known [10] that any two special spines of the same 3-manifold can be transformed into each other by a sequence of MP moves and their inverses provided that they have at least two vertices. Adding the Lune move the same fact holds without restrictions on the number of vertices.

The positive move MP has an obvious extension to the context of marked spines, because all old regions survive and we can define the new region not to be marked. This applies to the inverse of MP only if the triangular region that disappears with the move is not marked. Such an inverse MP-move is called admissible. It is then known [1] that any two o-spines of the same pair can be transformed into each other by a sequence of admissible MP-moves and their inverses provided that they have at least two vertices each.

The Lune move (see Fig. 4-right) has similar properties: whenever applied to a special spine of a manifold or to an o-spine of a pair, it yields a special spine of the same manifold or, respectively, an o-spine of the same pair. Application of an inverse Lune-move may lead to a non-special polyhedron and, just as an inverse MP-move, to the disappearance of a marked region. An inverse Lune-move is called admissible if neither of these happens. Any two o-spines of the same pair $(M, L)$, regardless of the number of vertices, are related by a sequence of admissible MP and Lune moves and their inverses.

Turaev-Viro initial data Let $\mathbb{K}$ be a commutative ring with unity. We recall that an initial datum for a Turaev-Viro invariant [19] consists of a finite set $I$, a function that assigns to each $i \in I$ its weight $w_{i} \in \mathbb{K}^{*}$, a certain distinguished element $w \in \mathbb{K}^{*}$, a distinguished set of unordered triples of elements of $I$, that are called admissible, and a function that assigns to each so-called admissible 6-tuple its $6 j$-symbol, which is an element of $\mathbb{K}$.

More precisely, an ordered 6-tuple $(i, j, k, l, m, n)$ of elements of $I$ is ad- 
missible if the unordered triples $(i, j, k),(i, m, n),(j, l, n),(k, l, m)$ are admissible. The $6 j$-symbol associated to this 6 -tuple is denoted by

$$
\left|\begin{array}{ccc}
i & j & k \\
l & m & n
\end{array}\right| .
$$

The symbols must have the following symmetries:

$$
\begin{aligned}
& \left|\begin{array}{ccc}
i & j & k \\
l & m & n
\end{array}\right|=\left|\begin{array}{ccc}
j & i & k \\
m & l & n
\end{array}\right|=\left|\begin{array}{ccc}
i & k & j \\
l & n & m
\end{array}\right| \\
= & \left|\begin{array}{ccc}
i & m & n \\
l & j & k
\end{array}\right|=\left|\begin{array}{ccc}
l & m & k \\
i & j & n
\end{array}\right|=\left|\begin{array}{ccc}
l & j & n \\
i & m & k
\end{array}\right| .
\end{aligned}
$$

Let us define some properties that an initial datum may (or not) satisfy. Notation and terminology come from the literature (see, for instance, [18] and references therein).

$(*)$ Orthogonality: For any $i^{\prime}, i^{\prime \prime}, j, k, l, n \in I$ such that the triples $\left(i^{\prime}, j, k\right)$, $\left(i^{\prime}, l, n\right),\left(i^{\prime \prime}, j, k\right),\left(i^{\prime \prime}, l, n\right)$ are admissible we have:

$$
\sum_{m} w_{m}\left|\begin{array}{ccc}
i^{\prime} & j & k \\
m & l & n
\end{array}\right|\left|\begin{array}{ccc}
i^{\prime \prime} & j & k \\
m & l & n
\end{array}\right|=w_{i}^{-1} \delta_{i^{\prime} i^{\prime \prime}}
$$

with sum taken over all $m$ such that both symbols are defined;

(**) Biedenharn-Elliot identity: For any admissible 6-tuples $\left(k, k^{\prime}, k^{\prime \prime}, j, j^{\prime}, j^{\prime \prime}\right)$ and $\left(k, k^{\prime}, k^{\prime \prime}, i, i^{\prime}, i^{\prime \prime}\right)$ we have:

$$
\begin{aligned}
& \left|\begin{array}{ccc}
k & k^{\prime} & k^{\prime \prime} \\
j & j^{\prime} & j^{\prime \prime}
\end{array}\right| \cdot\left|\begin{array}{ccc}
k & k^{\prime} & k^{\prime \prime} \\
i & i^{\prime} & i^{\prime \prime}
\end{array}\right| \\
= & \sum_{n} w_{n}\left|\begin{array}{ccc}
j^{\prime} & k & j^{\prime \prime} \\
i^{\prime \prime} & n & i^{\prime}
\end{array}\right| \cdot\left|\begin{array}{ccc}
j & k^{\prime} & j^{\prime \prime} \\
i^{\prime \prime} & n & i
\end{array}\right| \cdot\left|\begin{array}{ccc}
j & k^{\prime \prime} & j^{\prime} \\
i^{\prime} & n & i
\end{array}\right|
\end{aligned}
$$

with sum taken over all $n$ 's such that the involved symbols are defined;

$(* * *)$ For any $i \in I$ we have

$$
\sum_{j, k} w_{j} w_{k}=w \cdot w_{i}^{-1}
$$

with sum taken over all $j, k$ such that $(i, j, k)$ is admissible;

- Irreducibility: For any $j, k \in I$ there exists a sequence $l_{1}, l_{2}, \ldots, l_{n} \in I$ with $j=l_{1}$ and $k=l_{n}$ such that $\left(l_{i}, l_{i+1}, l_{i+2}\right)$ is admissible. 


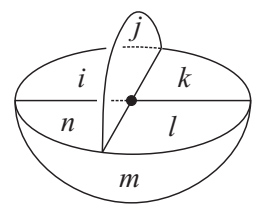

Figure 5: A coloring around a vertex yields a $6 j$-symbol

Turaev-Viro invariants To any irreducible initial datum satisfying Conditions $(*),(* *)$, and $(* * *)$ there corresponds a 3 -manifold invariant of Turaev-Viro type [19, Section 1.3], whose value on a given 3-manifold $M$ is obtained as follows. If $P$ is a special spine of $M$, we call a coloring of $P$ any map $\eta: R(P) \rightarrow I$. A coloring is called admissible if for any edge $e$ of $P$ the colors of the three germs of regions incident to $e$ form an admissible triple.

Denote the set of all admissible colorings of $P$ by $\operatorname{Adm}(P)$. If $\eta \in \operatorname{Adm}(P)$ and $v \in V(P)$, we define $s_{\eta}(v)$ as the $6 j$-symbol

$$
\left|\begin{array}{ccc}
i & j & k \\
l & m & n
\end{array}\right|
$$

where $i, j, k, l, m, n$ are the colors of the germs of regions of $P$ incident to $v$, as shown in Fig. 5. Note that $s_{\eta}(v)$ is well-defined because of the symmetries of the $6 j$-symbols.

We define the weight of $\eta \in \operatorname{Adm}(P)$ as

$$
\omega(\eta)=\prod_{v \in V(P)} s_{\eta}(v) \prod_{c \in R(P)} w_{\eta(c)}
$$

and the invariant of Turaev-Viro type corresponding to the initial datum as

$$
T V(M)=w^{-1} \cdot \sum_{\eta \in \operatorname{Adm}(P)} \omega(\eta) .
$$

As shown in [19], independence of the right-hand side of this equality from the particular choice of $P$ follows from Conditions $(*)$ and $(* *)$, which ensure invariance under Lune- and MP-moves and their inverses. Condition $(* * *)$ allows one to compute the invariant using a slightly more general notion of special spine. Namely, one requires $M \backslash P$ to be the union of some number $m \geqslant 1$ of balls, not necessarily a single one, and one replaces $w^{-1}$ in the formula defining $T V(M)$ by $w^{-m}$. 
Remark 1.5. The definition of the invariant also makes sense in the context of manifolds with boundary. Specifically, if $P$ is a special skeleton of a compact 3-manifold with boundary $M$ (i.e. if $P$ is special and $M \backslash P$ consists of the regular neighbourhood of the boundary and of some number $m$ of open balls) then the invariant of Turaev-Viro type of $M$ is again defined as $T V(M)=w^{-m} \sum_{\eta \in \operatorname{Adm}(P)} \omega(\eta)$. Notice also that this definition is different from that of the relative Turaev-Viro invariant $\Omega_{M}(\alpha)$ introduced in [19, Section 1.4].

\section{Turaev-Viro invariants of links}

In this section we describe certain additional requirements on the initial data and state the definition of colored Turaev-Viro invariants of links.

Good initial data The further conditions on the initial datum that we will need are the following ones.

- Strong irreducibility:

(i) For any $i, j \in I$ there exists $k \in I$ such that the triple $(i, j, k)$ is admissible;

(ii) For any two admissible triples of the form $(i, j, k)$ and $(i, m, n)$ there exist $l, l^{\prime} \in I$ such that the triples $(j, m, l),(k, n, l),\left(j, n, l^{\prime}\right)$, and $\left(k, m, l^{\prime}\right)$ are admissible;

(iii) For any two admissible 6 -tuples of the form $\left(i, j, k, i_{1}, k_{1}, j_{1}\right)$ and $\left(i, j, k, i_{2}, k_{2}, j_{2}\right)$ there exists $l$ such that all the triples $\left(i_{1}, i_{2}, l\right)$, $\left(j_{1}, j_{2}, l\right),\left(k_{1}, k_{2}, l\right)$ are admissible.

$(* * * *) \sum_{k} w_{k}=w_{i} w_{j}$ for any $i, j \in I$, with sum taken over all $k$ 's such that the triple $(i, j, k)$ is admissible.

An initial datum $\mathcal{D}$ is called good if it is strongly irreducible and it satisfies Conditions $(*),(* *),(* * *)$, and $(* * * *)$. 
Turaev-Viro invariants of colored links Let us fix a good initial datum. We will now define an invariant $\mathcal{I}_{\xi}(M, L)$ of triples $(M, L, \xi)$, where $\xi$ is a coloring of the link components by elements of $I$. Denote the components of $L$ by $L_{1}, \ldots, L_{n}$, so $\xi\left(L_{j}\right) \in I$ is the color of $L_{j}$. Let $P$ be an o-spine of $(M, L)$, let $\alpha_{j}$ be the region of $P$ dual to $L_{j}$, and let $m$ be the number of complementary balls having empty intersection with $L$. Denote by $\operatorname{Adm}_{\xi}(P)$ the set of all admissible colorings $\eta$ of $P$ such that $\eta\left(\alpha_{j}\right)=\xi\left(L_{j}\right)$ for all $j$. Then we set:

$$
\mathcal{I}_{\xi}(P)=w^{-m} \sum_{\eta \in \operatorname{Adm}_{\xi}(P)} \omega(\eta) .
$$

The next result is proved basically by the same argument as in [19] (see also [10]). See Section 4 for details.

Proposition 2.1. Let $P$ and $P^{\prime}$ be o-spines of a pair $(M, L)$. Then $\mathcal{I}_{\xi}(P)=$ $\mathcal{I}_{\xi}\left(P^{\prime}\right)$.

This proposition readily implies that we have a well-defined invariant $\mathcal{I}_{\xi}(M, L)$ of triples $(M, L, \xi)$ computed as $\mathcal{I}_{\xi}(P)$ whenever $P$ is an o-spine of $(M, L)$. It also follows from the definition that $\mathcal{I}(M, \emptyset)=T V(M)$ - there is no coloring $\xi$ to choose on the empty link.

Remark 2.2. As it will follow from the proof of Proposition 2.1, conditions $(*),(* *)$, and $(* * *)$ already imply that $\mathcal{I}_{\xi}(M, L)$ is well-defined. The other conditions imposed on good initial data will be used to analyze how $\mathcal{I}_{\xi}$ behaves under connected sums.

Remark 2.3. For the Turaev-Viro initial data [19, Section 7] the invariant $\mathcal{I}_{\xi}$ was already defined in 3 .

The invariants $\mathcal{I}_{i} \quad$ Fixing a color $i \in I$ we can define an invariant $\mathcal{I}_{i}(M, L)$ by coloring with $i$ all the components of $L$. When $M=\mathbb{S}^{3}$, we denote the invariant by $\mathcal{I}_{i}(L)$ only.

\section{General constructions}

In this section we describe several constructions that will be used extensively throughout the paper. 


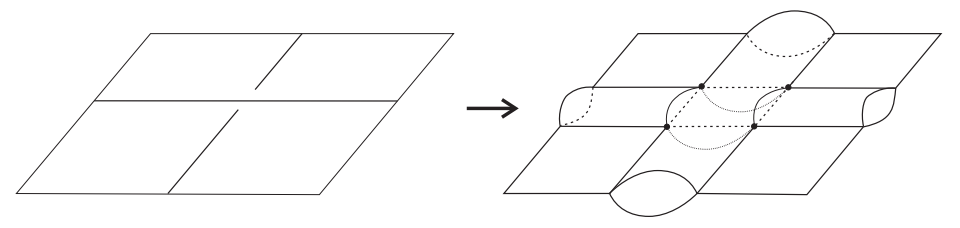

Figure 6: Digging the tunnel in the neighbourhood of a crossing point

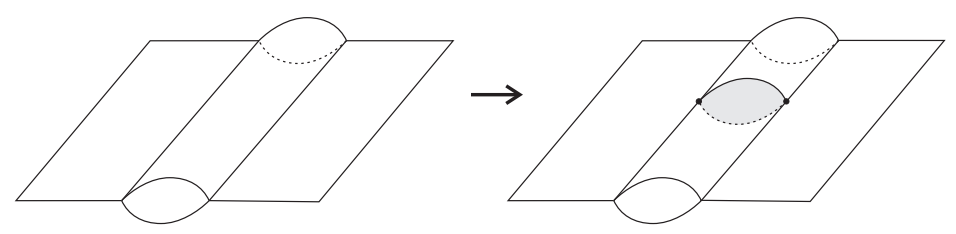

Figure 7: Gluing a transverse disc

An o-spine $P_{\Pi}(L)$ of $L \subset \mathbb{S}^{3}$ from a projection Given a connected projection $\Pi$ of a link $L \subset \mathbb{S}^{3}$ on a 2 -sphere $\mathbb{S}^{2} \subset \mathbb{S}^{3}$, we can always construct an o-spine of $L$ as follows. For each component of the link we "dig a tunnel" in $\mathbb{S}^{2}$ along the projection. Fig. [6 shows the way to do this in the neighbourhood of a crossing point. We get an embedded special polyhedron $P^{\prime}$ whose complement in $\mathbb{S}^{3}$ consists of two balls and a tubular neighbourhood $U(L)$ of the link. For each connected component of $L$ we now add a 2-disc to $P^{\prime}$ as in Fig. 7, denoting the resulting polyhedron by $P^{\prime \prime}$. Since $\mathbb{S}^{3} \backslash P^{\prime}$ consists, besides $U(L)$, of two balls, $P^{\prime \prime}$ is an o-spine of $\left(\mathbb{S}^{3}, L\right)$ : the marked discs are those of Fig. 7, and the orientation matters are easily settled. We then perform on $P^{\prime \prime}$ the move of Fig. 8 (on a single component of $L$ ); the result is still an o-spine of $\left(\mathbb{S}^{3}, L\right)$, but now the complement of $P^{\prime \prime}$ is just a regular neighborhood of $L$, without additional balls. We denote any polyhedron arising

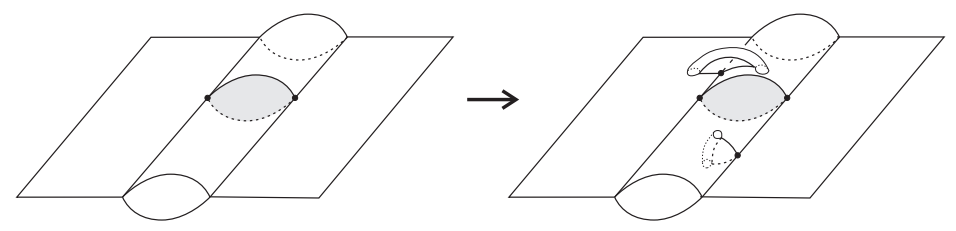

Figure 8: Getting rid of the extra balls in the complement 


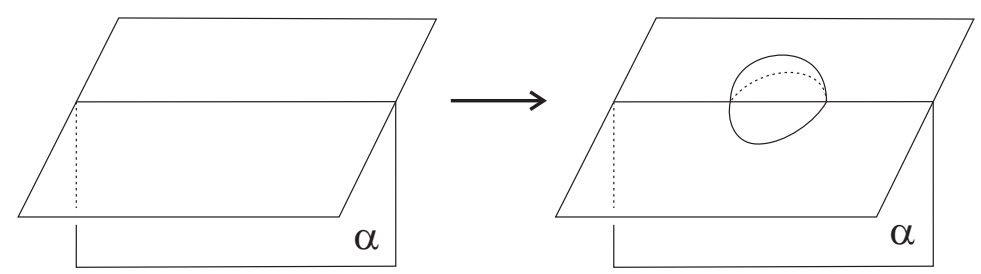

Figure 9: Adding a bubble

from this procedure by $P_{\Pi}(L)$.

A bubbled o-spine Let $P$ be a special polyhedron, and let $\alpha$ be a germ of region at an edge of $P$. A move as in Fig. 9 will be called addition of a bubble. The resulting polyhedron will be denoted by $\operatorname{Bub}_{\alpha}(P)$.

Join of polyhedra Let $P, Q$ be special polyhedra, let $\alpha$ be a germ of a region of $P$, and let $\beta$ be a germ of a region of $Q$. We then denote by Join $((P, \alpha),(Q, \beta))$ the special polyhedron which is obtained as in Fig. 10.

O-spine of an ordinary connected sum Let $P$ be an o-spine of a pair $\left(M_{1}, L_{1}\right)$, let $Q$ be an o-spine of $\left(M_{2}, L_{2}\right)$, and let $\alpha$ and $\beta$ be germs of regions of $P$ and of $Q$ respectively. Construct $\operatorname{Bub}_{\alpha}(P)$ first. We remark that $\alpha$ gives rise to three new germs in $\operatorname{Bub}_{\alpha}(P)$, exactly one of which is incident to both the two new vertices (this is the germ with boundary "inside the bubble", see Fig. 9). We denote this germ by $\bar{\alpha}$ and set

$$
\operatorname{OSum}_{\alpha, \beta}(P, Q):=\operatorname{Join}\left(\left(\operatorname{Bub}_{\alpha}(P), \bar{\alpha}\right),(Q, \beta)\right) .
$$

Lemma 3.1. The polyhedron $\operatorname{DSum}_{\alpha, \beta}(P, Q)$ is an o-spine of $\left(M_{1} \#_{o} M_{2}, L_{1} \sqcup\right.$ $\left.L_{2}\right)$.

The proof of this lemma is given by Fig. 11 (note that there may or not be components of $L_{1}$ and $L_{2}$ dual to $\alpha$ and $\beta$ ).

O-spine of a link-connected sum Let $P$ and $Q$ be o-spines of pairs $\left(M_{1}, L_{1}\right)$ and $\left(M_{2}, L_{2}\right)$. Let $K_{i}$ be a component of $L_{i}$, with $K_{1}$ dual to the region $\alpha$ of $P$ and $K_{2}$ dual to the region $\beta$ of $Q$. We construct first the marked oriented polyhedron $\operatorname{Join}\left(\left(\operatorname{Bub}_{\alpha}(P), \bar{\alpha}\right),(Q, \beta)\right)$ and denote by $c_{1}, \ldots, c_{4}$ the 


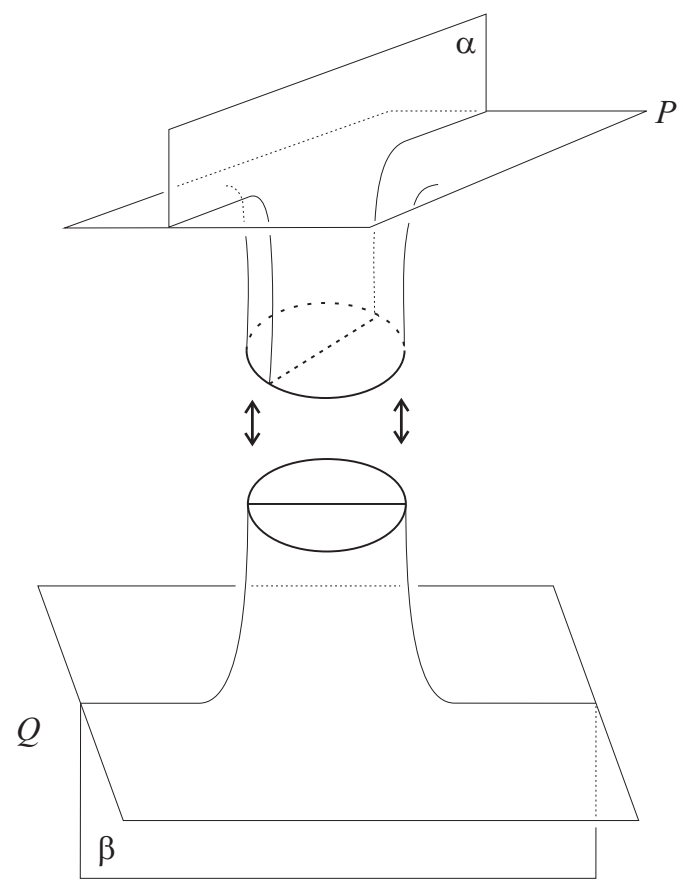

Figure 10: Construction of $\operatorname{Join}((P, \alpha),(Q, \beta))$ 

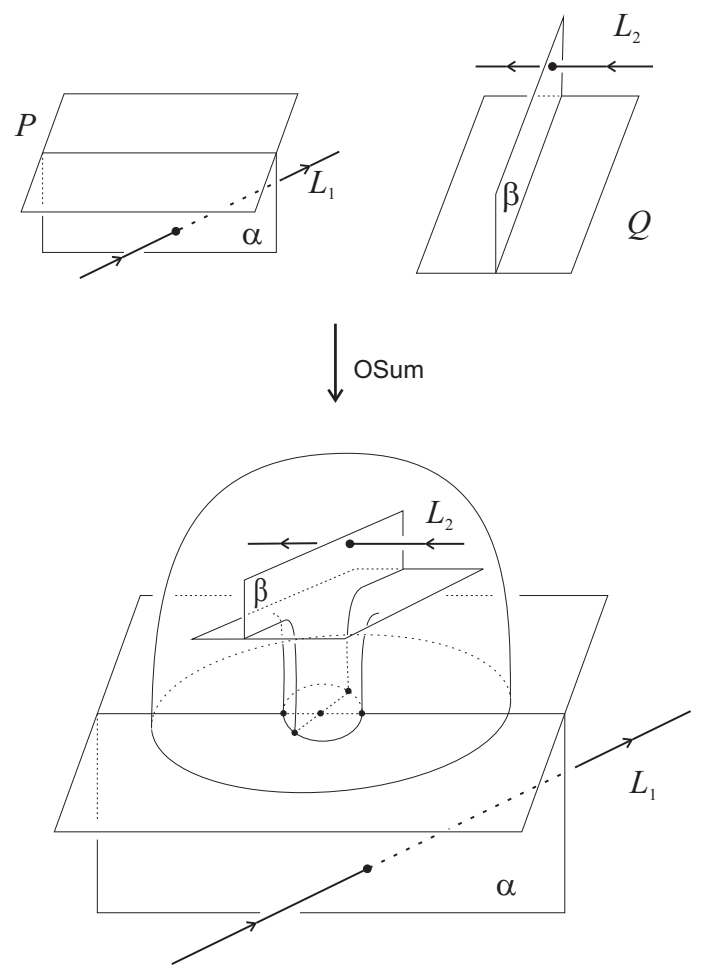

Figure 11: Construction of $\operatorname{OSum}_{\alpha, \beta}(P, Q)$ 


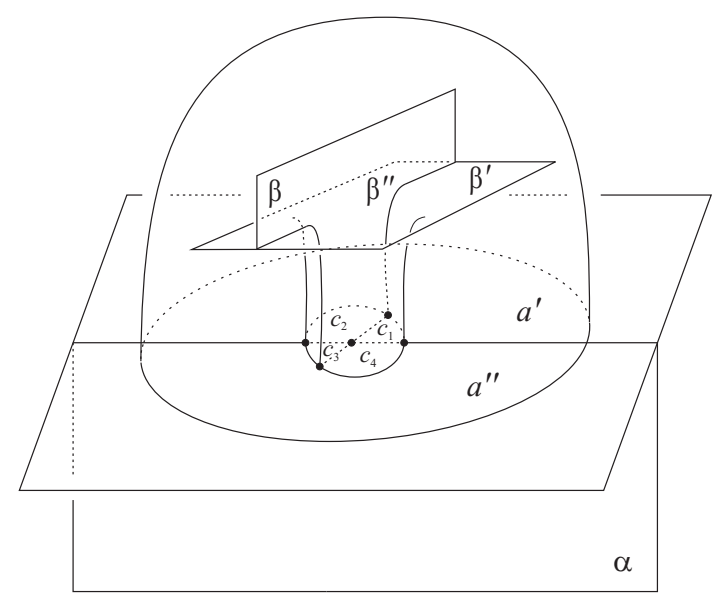

Figure 12: Notation for the regions of $\operatorname{Join}\left(\left(\operatorname{Bub}_{\alpha}(P), \bar{\alpha}\right),(Q, \beta)\right)$ used in the construction of $\operatorname{LSum}_{\alpha, \beta}(P, Q)$ and below in Section 4

four triangular regions that arise from identifying two discs in $\operatorname{Bub}_{\alpha}(P)$ and $Q$ (see Fig. 12).

For $i=1,2,3,4$ we denote by $\mu_{\alpha}\left(c_{i}\right)$ (respectively $\mu_{\beta}\left(c_{i}\right)$ ) the orientation of $c_{i}$ such that $\alpha$ (respectively $\beta$ ) and $c_{i}$ induce opposite orientations on their common edge. Then there are exactly two indices $s_{1}, s_{2} \in\{1,2,3,4\}$ such that $\mu_{\alpha}\left(c_{s_{j}}\right)=\mu_{\beta}\left(c_{s_{j}}\right)$. We then define the polyhedron $\operatorname{LSum}_{\alpha, \beta}(P, Q)$ as the o-spine obtained from $\operatorname{Join}\left(\left(\operatorname{Bub}_{\alpha}(P), \bar{\alpha}\right),(Q, \beta)\right)$ by removing the markings from $\alpha$ and $\beta$, placing the marking $\mu_{\alpha}\left(c_{i_{1}}\right)=\mu_{\beta}\left(c_{s_{1}}\right)$ on $c_{s_{1}}$, and removing the region $c_{s_{2}}$.

Lemma 3.2. Let $(M, L)$ be obtained by the link-connected sum of $\left(M_{1}, L_{1}\right)$ and $\left(M_{2}, L_{2}\right)$ along $K_{1}$ and $K_{2}$. Then any $\operatorname{LSum}_{\alpha, \beta}(P, Q)$ constructed as above is an o-spine of $(M, L)$.

The proof of this lemma is given by Fig. 13, where we assume $s_{1}=3$ and $s_{2}=1$.

\section{State-sums with good initial data}

In this section we describe the behaviour of $\mathcal{I}_{\xi}$ under the operations Bub, Join, OSum, and LSum. We then apply these results to discuss how the invariants behave under ordinary and link-connected sum of pairs. 

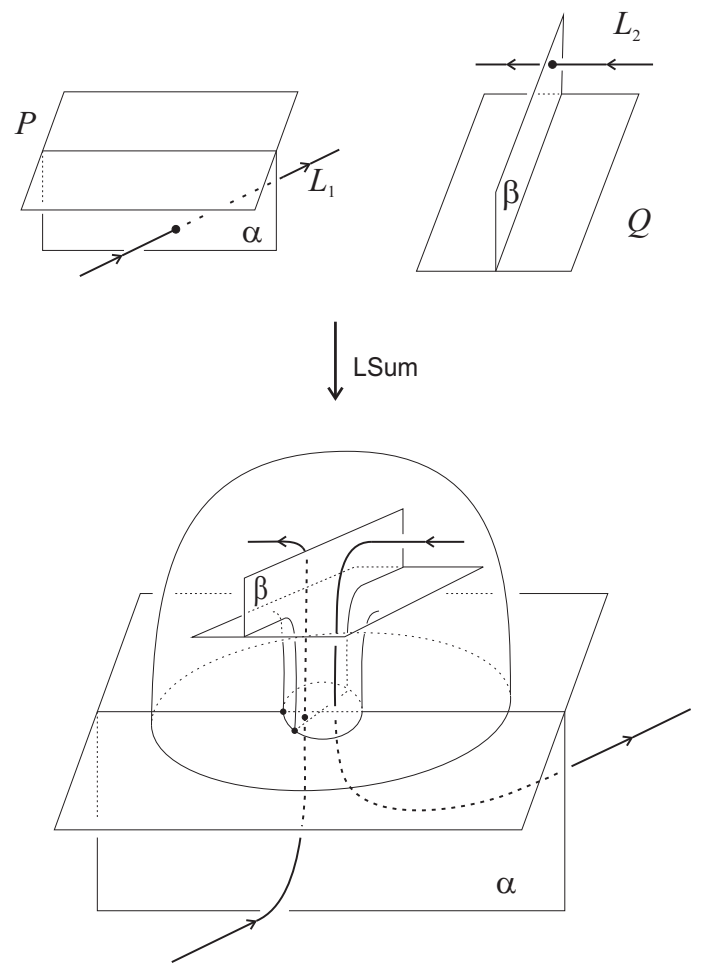

Figure 13: Construction of $\operatorname{LSum}_{\alpha, \beta}(P, Q)$ 


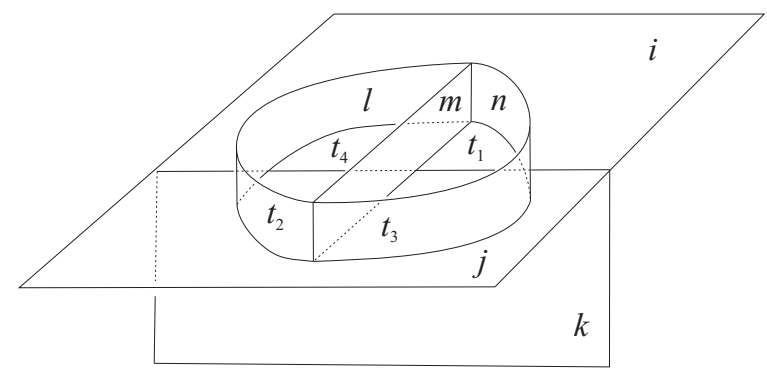

Figure 14: The polyhedron $P^{\prime}$

Some notation The following notation will be handy. If $\alpha$ is a region of a special polyhedron $P$ and $i$ is a color, we denote by $\operatorname{Adm}_{(\alpha, i)}(P)$ the set of all admissible colorings of $P$ where $\alpha$ is colored by $i$. Furthermore, if $\xi_{1}$ and $\xi_{2}$ are colorings of disjoint lists of regions of $P$, we denote their union simply by $\xi_{1}, \xi_{2}$. Let now $P$ be an o-spine of a pair $(M, L)$, let $\alpha_{1}, \ldots, \alpha_{n}$ be a list of regions of $P$ (that may or may not be marked), and let $i_{1}, \ldots, i_{n}$ be colors. Then for any coloring $\xi$ of the components of $L$ that are not dual to any of $\alpha_{i}$, we have

$$
\mathcal{I}_{\xi,\left(\alpha_{1}, i_{1}\right), \ldots,\left(\alpha_{n}, i_{n}\right)}(P)=w^{-m} \sum_{\eta \in \operatorname{Adm}_{\xi,\left(\alpha_{1}, i_{1}\right), \ldots,\left(\alpha_{n}, i_{n}\right)}(P)} \omega(\eta) .
$$

where $m$ is the number of complementary balls having empty intersection with $L$.

Some properties of good initial data The first property that we prove does not in fact require Conditions $(*)-(* * * *)$.

Lemma 4.1. For any choice of a strongly irreducible datum and for any two admissible triples $(i, j, k)$ and $(l, m, n)$ there exist $t_{1}, t_{2}, t_{3}, t_{4} \in I$ such that the coloring of the polyhedron $P^{\prime}$ shown in Fig. 14 is admissible.

Proof. Denote by $P^{\prime \prime}$ the polyhedron obtained from $P^{\prime}$ by the inverse MPmove along the region colored by $t_{1}$. By Condition (iii) of strong irreducibility the desired $t_{1}, t_{2}, t_{3}, t_{4}$ exist if and only if there exist $t_{2}, t_{3}, t_{4}$ such that the resulting coloring on $P^{\prime \prime}$ is admissible. Notice that the neighborhoods in $P^{\prime \prime}$ of the regions colored by $t_{2}, t_{3}, t_{4}$ have the form shown in Fig. 15, By 

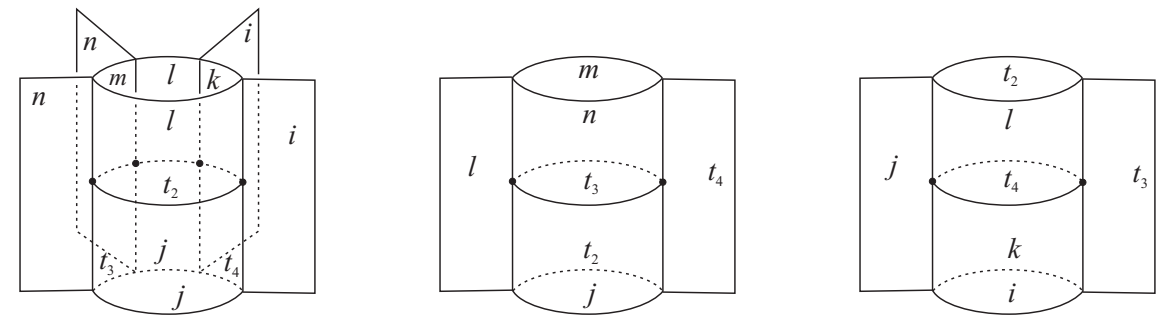

Figure 15: Neighbourhoods of regions in $P^{\prime \prime}$

Condition (i) of strong irreducibility we can find $t_{2}$ such that the triple $\left(l, j, t_{2}\right)$ is admissible. Since $(l, m, n)$ is admissible by assumption, by Condition (ii) of strong irreducibility we can find a $t_{3}$ such that both of the triples $\left(j, n, t_{3}\right)$ and $\left(m, t_{2}, t_{3}\right)$ are admissible. Similarly, since $(j, i, k)$ and $\left(l, j, t_{2}\right)$ are admissible, we can find $t_{4}$ such that $\left(i, l, t_{4}\right)$ and $\left(k, t_{2}, t_{4}\right)$ are both admissible, whence the conclusion.

For the rest of this section we now fix some good initial datum.

Lemma 4.2. Let $\left(i, i^{\prime}, i^{\prime \prime}\right)$ and $\left(j, j^{\prime}, j^{\prime \prime}\right)$ be admissible triples of colors. Then

$$
\begin{aligned}
\sum_{k, l, m, n} w_{k} w_{l} w_{m} w_{n}\left|\begin{array}{ccc}
i^{\prime \prime} & j^{\prime} & l \\
j & k & j^{\prime \prime}
\end{array}\right| \cdot\left|\begin{array}{ccc}
i^{\prime \prime} & j^{\prime} & l \\
m & i & i^{\prime}
\end{array}\right| \\
\times\left|\begin{array}{ccc}
i & i^{\prime \prime} & i^{\prime} \\
j^{\prime \prime} & n & k
\end{array}\right| \cdot\left|\begin{array}{ccc}
m & j^{\prime} & i^{\prime} \\
j^{\prime \prime} & n & j
\end{array}\right| \cdot\left|\begin{array}{ccc}
i & l & m \\
j & n & k
\end{array}\right|=1
\end{aligned}
$$

with sum taken over all $k, l, m, n$ such that the involved symbols are defined.

Proof. Collecting together all the summands containing $n$ and applying (**), we conclude that that the expression we want to evaluate is equal to

$$
\begin{aligned}
& \sum_{k, l, m} w_{k} w_{l} w_{m}\left|\begin{array}{ccc}
i^{\prime \prime} & j^{\prime} & l \\
j & k & j^{\prime \prime}
\end{array}\right|^{2} \cdot\left|\begin{array}{ccc}
i^{\prime \prime} & j^{\prime} & l \\
m & i & i^{\prime}
\end{array}\right|^{2} \\
= & \sum_{k, l} w_{k} w_{l}\left|\begin{array}{ccc}
i^{\prime \prime} & j^{\prime} & l \\
j & k & j^{\prime \prime}
\end{array}\right|^{2} \cdot\left(\sum_{m} w_{m}\left|\begin{array}{ccc}
i^{\prime \prime} & j^{\prime} & l \\
m & i & i^{\prime}
\end{array}\right|^{2}\right) \\
= & \frac{1}{w_{i^{\prime \prime}}} \sum_{k, l} w_{k} w_{l}\left|\begin{array}{ccc}
i^{\prime \prime} & j^{\prime} & l \\
j & k & j^{\prime \prime}
\end{array}\right|^{2}=\sum_{k} \frac{w_{k}}{w_{i^{\prime \prime}} w_{j^{\prime \prime}}}
\end{aligned}
$$




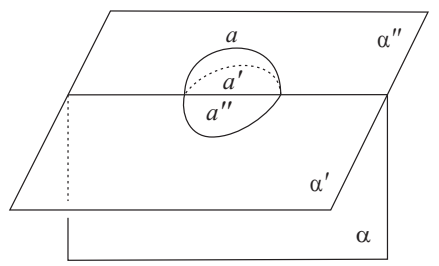

Figure 16: Notation for the regions of a bubbled polyhedron

where we have used $(*)$ twice and the last sum is taken over all $k$ such that $\left(i^{\prime \prime}, j^{\prime \prime}, k\right)$ is admissible. By Condition $(* * * *)$ we get the conclusion.

State-sums on bubbled polyhedra Here we investigate the behaviour of state-sums with good initial data under the bubble move. The lemmas below use the notation of Fig. 16.

Lemma 4.3. Let $P$ be an o-spine, and let $\alpha$ be a region of $P$. Let $\xi$ be a coloring of all marked regions of $P$ except $\alpha, \alpha^{\prime}, \alpha^{\prime \prime}$ if any of them is marked. Then for any $i, i^{\prime}, i^{\prime \prime}, j, k \in I$ such that the triples $\left(i, i^{\prime}, i^{\prime \prime}\right)$ and $(i, j, k)$ are admissible, we have the equality

$$
\mathcal{I}_{\xi,(\alpha, i),\left(\alpha^{\prime}, i^{\prime}\right),\left(\alpha^{\prime \prime}, i^{\prime \prime}\right),\left(a^{\prime}, j\right),\left(a^{\prime \prime}, k\right)}\left(\operatorname{Bub}_{\alpha}(P)\right)=w^{-1} \frac{w_{j} w_{k}}{w_{i}} \mathcal{I}_{\xi,(\alpha, i),\left(\alpha^{\prime}, i^{\prime}\right),\left(\alpha^{\prime \prime}, i^{\prime \prime}\right)}(P) .
$$

Proof. Notice that any coloring in $\operatorname{Adm}_{\xi,(\alpha, i),\left(\alpha^{\prime}, i^{\prime}\right),\left(\alpha^{\prime \prime}, i^{\prime \prime}\right),\left(a^{\prime}, j\right),\left(a^{\prime \prime}, k\right)}\left(\operatorname{Bub}_{\alpha}(P)\right)$ naturally induces a coloring in $\operatorname{Adm}_{\xi,(\alpha, i),\left(\alpha^{\prime}, i^{\prime}\right),\left(\alpha^{\prime \prime}, i^{\prime \prime}\right)}(P)$, and the resulting map

$$
\operatorname{Adm}_{\xi,(\alpha, i),\left(\alpha^{\prime}, i^{\prime}\right),\left(\alpha^{\prime \prime}, i^{\prime \prime}\right),\left(a^{\prime}, j\right),\left(a^{\prime \prime}, k\right)}\left(\operatorname{Bub}_{\alpha}(P)\right) \rightarrow \operatorname{Adm}_{\xi,(\alpha, i),\left(\alpha^{\prime}, i^{\prime}\right),\left(\alpha^{\prime \prime}, i^{\prime \prime}\right)}(P)
$$

is surjective (this follows from Condition (ii) of strong irreducibility). Notice further that the complement of $\operatorname{Bub}_{\alpha}(P)$ contains one more empty ball with respect to $P$. Let $l$ denote the (varying) color of $a$. By the above remarks we have

$$
\begin{aligned}
& \mathcal{I}_{\xi,(\alpha, i),\left(\alpha^{\prime}, i^{\prime}\right),\left(\alpha^{\prime \prime}, i^{\prime \prime}\right),\left(a^{\prime}, j\right),\left(a^{\prime \prime}, k\right)}\left(\operatorname{Bub}_{\alpha}(P)\right) \\
& =w^{-1} \cdot w_{j} w_{k} \cdot\left(\sum_{l} w_{l}\left|\begin{array}{ccc}
i & i^{\prime} & i^{\prime \prime} \\
l & j & k
\end{array}\right|^{2}\right) \cdot \mathcal{I}_{\xi,(\alpha, i),\left(\alpha^{\prime}, i^{\prime}\right),\left(\alpha^{\prime \prime}, i^{\prime \prime}\right)}(P) .
\end{aligned}
$$

By Condition $(*)$ the sum between brackets in this expression evaluates to $w_{i}^{-1}$, whence the conclusion. 
Corollary 4.4. Under the assumptions of the lemma, we have

$$
\mathcal{I}_{\xi,(\alpha, i),\left(a^{\prime}, j\right),\left(a^{\prime \prime}, k\right)}\left(\operatorname{Bub}_{\alpha}(P)\right)=w^{-1} \frac{w_{j} w_{k}}{w_{i}} \mathcal{I}_{\xi,(\alpha, i)}(P) .
$$

Lemma 4.5. Let $P$ be an o-spine, and let $\alpha$ be a region of $P$. Then for any choice of a coloring $\xi$ of the marked regions of $P$ we have

$$
\mathcal{I}_{\xi}\left(\operatorname{Bub}_{\alpha}(P)\right)=\mathcal{I}_{\xi}(P) .
$$

Proof. If $\alpha$ is not marked then by Corollary 4.4 we have that

$$
\mathcal{I}_{\xi}\left(\operatorname{Bub}_{\alpha}(P)\right)=w^{-1} \cdot \sum_{i, j, k} \frac{w_{j} w_{k}}{w_{i}} \mathcal{I}_{\xi,(\alpha, i)}(P)
$$

with sum taken over all $i, j, k$ such that the triple $(i, j, k)$ is admissible, and the conclusion follows from Condition $(* * *)$ together with the obvious equality $\mathcal{I}_{\xi}(P)=\sum_{i} \mathcal{I}_{\xi,(\alpha, i)}(P)$. If $\alpha$ is marked and $\xi^{\prime}$ is the restriction of $\xi$ to all the marked regions of $P$ other than $\alpha$ then again by Corollary 4.4 we have that

$$
\mathcal{I}_{\xi}\left(\operatorname{Bub}_{\alpha}(P)\right)=w^{-1} \cdot \sum_{j, k} \frac{w_{j} w_{k}}{w_{i}} \mathcal{I}_{\xi^{\prime},(\alpha, i)}(P)=w^{-1} \cdot \sum_{j, k} \frac{w_{j} w_{k}}{w_{i}} \mathcal{I}_{\xi}(P),
$$

and the conclusion again follows from Condition $(* * *)$.

Using this lemma we can now spell out the proof of the fact that colored Turaev-Viro invariants for links are well-defined under our assumption that the initial data are good:

Proof of [2.1. It follows from [1, Theorem 2.1, Corollary 2.2] that there exists a sequence of bubble moves and of admissible Lune- and MP-moves and their inverses taking $P$ to $P^{\prime}$. The conclusion easily follows, because Lemma 4.5 implies invariance of the colored state-sum under bubble moves, whereas, just as in [19, 10], $(*)$ implies invariance under Lune and its inverse, and $(* *)$ implies invariance under MP and its inverse.

State-sums on joins We now turn to the behaviour of the invariants under joins of o-spines. We will in fact consider a somewhat specific situation, which will suffice for our purposes. Namely, given a germ $\alpha$ of a region along an edge we consider the three germs of the complementary balls whose closures have 


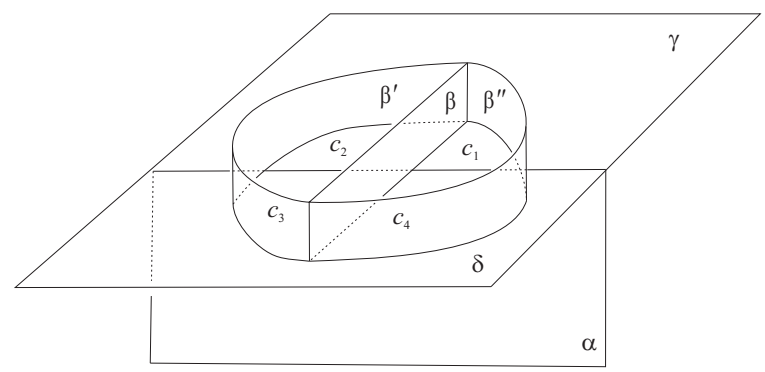

Figure 17: Notation for the regions of a join

non-empty intersection with $\alpha$. The closures of two of these germs contain the whole of $\alpha$, while the closure of the third one intersects it only along a segment. We will call the ball containing this third germ the complementary ball opposite to the germ $\alpha$.

Proposition 4.6. Let $P$ and $Q$ be o-spines, let $\alpha$ be a germ of a region of $P$ such that the complementary ball opposite to $\alpha$ is empty, and let $\beta$ be a germ of a region of $Q$. Let us fix a coloring $\xi_{1}$ of the marked regions of $P$ and a coloring $\xi_{2}$ of the marked regions of $Q$. Then

$$
\mathcal{I}_{\xi_{1}, \xi_{2}}(\operatorname{Join}((P, \alpha),(Q, \beta)))=w \cdot \mathcal{I}_{\xi_{1}}(P) \cdot \mathcal{I}_{\xi_{2}}(Q) .
$$

Proof. Let $m_{1}$ be the number of empty complementary balls of $P$, and let $m_{2}$ be the number of empty complementary balls of $Q$. By the assumptions of the lemma, the number of empty complementary balls of Join $((P, \alpha),(Q, \beta))$ is equal to $m_{1}+m_{2}-1$. Furthermore, since an admissible coloring of Join $((P, \alpha),(Q, \beta))$ induces admissible colorings on $P$ and on $Q$, there is a naturally defined map

$$
\operatorname{Adm}_{\xi_{1}, \xi_{2}}(\operatorname{Join}((P, \alpha),(Q, \beta))) \rightarrow \operatorname{Adm}_{\xi_{1}}(P) \times \operatorname{Adm}_{\xi_{2}}(Q),
$$

and by Lemma 4.1 this map is surjective. Using the notation of Fig. 17 and 
agreeing that $t_{i}$ will denote the color of $c_{i}$ for $i=1, \ldots, 4$, we see that

$$
\begin{gathered}
\mathcal{I}_{\xi_{1}, \xi_{2}}(\operatorname{Join}((P, \alpha),(Q, \beta)))=w \cdot w^{-m_{1}-m_{2}} \sum_{\left(\eta_{1}, \eta_{2}\right)} \omega\left(\eta_{1}\right) \cdot \omega\left(\eta_{2}\right) \\
\times\left(\sum_{t_{1}, t_{2}, t_{3}, t_{4}} w_{t_{1}} w_{t_{2}} w_{t_{3}} w_{t_{4}}\left|\begin{array}{ccc}
i^{\prime \prime} & j^{\prime} & t_{2} \\
j & t_{1} & j^{\prime \prime}
\end{array}\right| \cdot\left|\begin{array}{ccc}
i^{\prime \prime} & j^{\prime} & t_{2} \\
t_{3} & i & i^{\prime}
\end{array}\right|\right. \\
\left.\quad \cdot\left|\begin{array}{ccc}
i & i^{\prime \prime} & i^{\prime} \\
j^{\prime \prime} & t_{4} & t_{1}
\end{array}\right| \cdot\left|\begin{array}{ccc}
t_{3} & j^{\prime} & i^{\prime} \\
j^{\prime \prime} & t_{4} & j
\end{array}\right| \cdot\left|\begin{array}{ccc}
i & t_{2} & t_{3} \\
j & t_{4} & t_{1}
\end{array}\right|\right),
\end{gathered}
$$

where $\left(\eta_{1}, \eta_{2}\right)$ runs over the set $\operatorname{Adm}_{\xi_{1}}(P) \times \operatorname{Adm}_{\xi_{2}}(Q)$, the colors $t_{i}$ are such that the involved symbols are defined, and we write for brevity $i=\eta_{1}(\alpha)$, $i^{\prime}=\eta_{1}(\delta), i^{\prime \prime}=\eta_{1}(\gamma), j=\eta_{2}(\beta), j^{\prime}=\eta_{2}\left(\beta^{\prime}\right), j^{\prime \prime}=\eta_{2}\left(\beta^{\prime \prime}\right)$. By Lemma 4.2 the sum between brackets in this expression always evaluates to 1 , whence the conclusion.

Recall that the set of marked regions of the polyhedron $\operatorname{LSum}_{\alpha, \beta}(P, Q)$ consists of the set of marked regions of $P$ minus $\alpha$, the set of marked regions of $Q$ minus $\beta$, and a new region $c_{s_{1}}$, the definition of which was given immediately before Lemma 3.2 .

Proposition 4.7. Let $P$ and $Q$ be o-spines, let $\alpha$ be a marked region of $P$, and let $\beta$ be a marked region of $Q$. Let $\xi_{1}$ be a coloring of all the marked regions of $P$ other than $\alpha$, and let $\xi_{2}$ be a coloring of all the marked regions of $Q$ other than $\beta$. Let $k$ be a color. Then

$$
\mathcal{I}_{\xi_{1}, \xi_{2},\left(c_{s_{1}}, k\right)}\left(\operatorname{LSum}_{\alpha, \beta}(P, Q)\right)=\sum_{i, j} \frac{w_{k}}{w_{i} w_{j}} \mathcal{I}_{\xi_{1},(\alpha, i)}(P) \cdot \mathcal{I}_{\xi_{2},(\beta, j)}(Q)
$$

with sum taken over all colors $i, j$ such that the triple $(i, j, k)$ is admissible.

Proof. Without loss of generality we may assume that $s_{1}=1$ in the notation of Fig. 12. Then $s_{2}=3$. Recall that $\operatorname{LSum}_{\alpha, \beta}(P, Q)$ is obtained from a certain Join $\left(\left(\operatorname{Bub}_{\alpha}(P), \bar{\alpha}\right),(Q, \beta)\right)$ by removing the region $c_{3}$. As a consequence of this removal, the regions $c_{2}, c_{4}$, and $a^{\prime \prime}$ get absorbed into $\alpha, \beta$, and $\beta^{\prime \prime}$, respectively. Thus, an admissible coloring of $\operatorname{LSum}_{\alpha, \beta}(P, Q)$ induces admissible colorings on $\operatorname{Bub}_{\alpha}(P)$ and on $Q$, and the resulting map of colorings

$$
\begin{aligned}
\operatorname{Adm}_{\xi_{1}, \xi_{2},\left(c_{1}, k\right),(\alpha, i),\left(a^{\prime}, i^{\prime}\right),(\beta, j),\left(\beta^{\prime}, j^{\prime}\right),\left(\beta^{\prime \prime}, j^{\prime \prime}\right)}\left(\operatorname{LSum}_{\alpha, \beta}(P, Q)\right) & \\
\longrightarrow \operatorname{Adm}_{\xi_{1},(\alpha, i),\left(a^{\prime}, i^{\prime}\right),\left(b, j^{\prime}\right)}\left(\operatorname{Bub}_{\alpha}(P)\right) \times \operatorname{Adm}_{\xi_{2},(\beta, j),\left(\beta^{\prime}, j^{\prime}\right),\left(\beta^{\prime \prime}, j^{\prime \prime}\right)}(Q) &
\end{aligned}
$$


is onto for any $i, j, i^{\prime}, j^{\prime}, j^{\prime \prime}$ such that the triples $(i, j, k),\left(i^{\prime}, j^{\prime}, k\right)$, and $\left(i, i^{\prime}, j^{\prime \prime}\right)$ are admissible.

Denoting the varying color of $\alpha=c_{2}$ by $i$, the color of $a^{\prime}$ by $i^{\prime}$, the color of $\beta=c_{4}$ by $j$, the color of $\beta^{\prime}$ by $j^{\prime}$, and the color of $\beta^{\prime \prime}=a^{\prime \prime}$ by $j^{\prime \prime}$, from the construction of $\operatorname{LSum}_{\alpha, \beta}(P, Q)$ we conclude that

$$
\begin{aligned}
\mathcal{I}_{\xi_{1}, \xi_{2},\left(c_{1}, k\right)}\left(\operatorname{LSum}_{\alpha, \beta}(P, Q)\right) & =\sum_{i, j} \sum_{j^{\prime}, j^{\prime \prime}} \sum_{i^{\prime}} w \cdot \mathcal{I}_{\xi_{1},(\alpha, i),\left(a^{\prime}, i^{\prime}\right),\left(a^{\prime \prime}, j^{\prime \prime}\right)}\left(\operatorname{Bub}_{\alpha}(P)\right) \\
& \times \mathcal{I}_{\xi_{2},(\beta, j),\left(\beta^{\prime}, j^{\prime}\right),\left(\beta^{\prime \prime}, j^{\prime \prime}\right)}(Q) \cdot w_{k} w_{j^{\prime \prime}}^{-1}\left|\begin{array}{ccc}
k & i & j \\
j^{\prime \prime} & j^{\prime} & i^{\prime}
\end{array}\right|^{2}
\end{aligned}
$$

with sum taken over all indices such that the symbol is defined. By Corollary 4.4 this is equal to

$$
\sum_{i, j} \mathcal{I}_{\xi_{1},(\alpha, i)}(P) \cdot \sum_{j^{\prime}, j^{\prime \prime}} \mathcal{I}_{\xi_{2},(\beta, j),\left(\beta^{\prime}, j^{\prime}\right),\left(\beta^{\prime \prime}, j^{\prime \prime}\right)}(Q) \cdot w_{k} w_{i}^{-1} \cdot\left(\sum_{i^{\prime}} w_{i^{\prime}}\left|\begin{array}{ccc}
k & i & j \\
j^{\prime \prime} & j^{\prime} & i^{\prime}
\end{array}\right|^{2}\right) \text {. }
$$

Using Condition (*), we get

$$
\begin{aligned}
\sum_{i, j} \mathcal{I}_{\xi_{1},(\alpha, i)}(P) w_{k} w_{i}^{-1} w_{j}^{-1} \cdot \sum_{j^{\prime}, j^{\prime \prime}} \mathcal{I}_{\xi_{2},(\beta, j),\left(\beta^{\prime}, j^{\prime}\right),\left(\beta^{\prime \prime}, j^{\prime \prime}\right)}(Q) & \\
& =\sum_{i, j} \frac{w_{k}}{w_{i} w_{j}} \mathcal{I}_{\xi_{1},(\alpha, i)}(P) \cdot \mathcal{I}_{\xi_{2},(\beta, j)}(Q),
\end{aligned}
$$

whence the conclusion.

Behaviour under connected sum The above results now easily allow us to describe the behaviour of the invariants under connected sum. For the ordinary version, Proposition 4.6 and Lemma 4.5 immediately imply:

Theorem 4.8. Let $\xi_{t}$ be a coloring of a link $L_{t} \subset M_{t}$ for $t=1,2$. Then

$$
\mathcal{I}_{\xi_{1}, \xi_{2}}\left(M_{1} \#_{o} M_{2}, L_{1} \sqcup L_{2}\right)=w \cdot \mathcal{I}_{\xi_{1}}\left(M_{1}, L_{1}\right) \cdot \mathcal{I}_{\xi_{2}}\left(M_{2}, L_{2}\right) .
$$

Let us now turn to the link-connected sum. Let $K_{t}$ be a connected component of a link $L_{t} \subset M_{t}$, let $\xi_{t}$ be a coloring of $L_{t} \backslash K_{t}$ for $t=1,2$, and let $(M, L)$ be the link-connected sum of $\left(M_{1}, L_{1}\right)$ and $\left(M_{2}, L_{2}\right)$ along $K_{1}$ and $K_{2}$. If $\xi$ is one of the colorings $\xi_{1}, \xi_{2}$, or $\xi_{1}, \xi_{2}$ then there is one component of the relevant link not colored by $\xi$, and we define the coloring $(\xi ; i)$ by giving it color $i$. Proposition 4.7 implies: 
Theorem 4.9. We have

$$
\frac{1}{w_{k}} \mathcal{I}_{\left(\xi_{1}, \xi_{2} ; k\right)}(M, L)=\sum_{i, j} \frac{1}{w_{i}} \mathcal{I}_{\left(\xi_{1} ; i\right)}\left(M_{1}, L_{1}\right) \cdot \frac{1}{w_{j}} \mathcal{I}_{\left(\xi_{2} ; j\right)}\left(M_{2}, L_{2}\right)
$$

with sum taken over all $i, j$ such that the triple $(i, j, k)$ is admissible.

In the case of knots the above formula has a particularly nice form:

$$
\frac{1}{w_{k}} \mathcal{I}_{k}\left(M_{1} \# M_{2}, K_{1} \# K_{2}\right)=\sum_{i, j} \frac{1}{w_{i}} \mathcal{I}_{i}\left(M_{1}, K_{1}\right) \cdot \frac{1}{w_{j}} \mathcal{I}_{j}\left(M_{2}, K_{2}\right) .
$$

\section{The $\varepsilon$-invariant}

We now specialize our analysis of colored Turaev-Viro invariants to the case of the good initial data of [9]. Another specialization will be discussed in the next section.

Definition The so-called $\varepsilon$-invariant of 3-manifolds was introduced in [9] (see also [10, Section 8.1.3]). Let us fix a root $\varepsilon$ of the equation $x^{2}=x+1$, and an arbitrary square root $\varepsilon^{1 / 2}$ of $\varepsilon$. The corresponding initial datum $\mathcal{D}_{\varepsilon}$ consists of the following. The ring $\mathbb{K}$ is $\mathbb{C}$. The set $I$ is the set $\{0,1\}$. The weights are given by $w_{0}=1, w_{1}=\varepsilon$, and the constant $w$ is equal to $\varepsilon+2$. The admissible triples are those satisfying the so-called triangle inequalities: a triple $(i, j, k)$ is admissible if and only if

$$
i \leqslant j+k, \quad j \leqslant k+i, \quad k \leqslant i+j .
$$

The values of the $6 j$-symbols are given by:

$$
\begin{aligned}
& \left|\begin{array}{lll}
0 & 0 & 0 \\
0 & 0 & 0
\end{array}\right|_{\varepsilon}=1,\left|\begin{array}{lll}
0 & 0 & 0 \\
1 & 1 & 1
\end{array}\right|_{\varepsilon}=\varepsilon^{-1 / 2}, \\
& \left|\begin{array}{lll}
0 & 1 & 1 \\
0 & 1 & 1
\end{array}\right|_{\varepsilon}=\varepsilon^{-1},\left|\begin{array}{lll}
0 & 1 & 1 \\
1 & 1 & 1
\end{array}\right|_{\varepsilon}=\varepsilon^{-1},\left|\begin{array}{lll}
1 & 1 & 1 \\
1 & 1 & 1
\end{array}\right|_{\varepsilon}=-\varepsilon^{-2} .
\end{aligned}
$$

Proposition 5.1. The datum just described is good.

Proof. Strong irreducibility and Condition $(* * * *)$ can be checked directly. Condition $(* *)$ was established in [10, Section 8.1.3]. 
Let us prove that the datum satisfies $(* * *)$. If $k=0$ then the sum on the left-hand side of $(* * *)$ is equal to $w_{0}^{2}+w_{1}^{2}=\varepsilon+2=(\varepsilon+2) w_{0}$. If $k=1$ then the sum is equal to $w_{0} w_{1}+w_{1} w_{0}+w_{1}^{2}=(\varepsilon+2) \varepsilon=w \cdot w_{1}$, whence the conclusion.

To prove orthogonality, we consider various cases. If $i=i^{\prime}=0$, we must evaluate

$$
\sum_{m} w_{m}\left|\begin{array}{ccc}
0 & j & j \\
m & l & l
\end{array}\right|_{\varepsilon}^{2}
$$

where up to symmetry we can assume that $(j, l)$ is $(0,0),(1,0)$, or $(1,1)$, and a simple calculation shows that the sum is always 1 , whence the desired conclusion.

Suppose now that $i=i^{\prime}=1$. Then up to symmetry $(j, k)$ is either $(1,0)$ or $(1,1)$. If $(j, k)=(1,0)$ then $m=l$, so we should evaluate the expression

$$
w_{l}\left|\begin{array}{lll}
1 & 1 & 0 \\
l & l & n
\end{array}\right|_{\varepsilon}^{2}
$$

for $l, n$ not simultaneously zero. In all cases we get $\varepsilon^{-1}$, i.e. $w_{1}^{-1}$. Suppose now that $(j, k)=(1,1)$. If either $l$ or $n$ is zero, the value of the expression is

$$
w_{1}\left|\begin{array}{lll}
1 & 1 & 1 \\
1 & 1 & 0
\end{array}\right|_{\varepsilon}^{2}=\varepsilon^{-1}=w_{1}^{-1}
$$

while if also $l=n=1$, it is

$$
w_{0}\left|\begin{array}{ccc}
1 & 1 & 1 \\
0 & 1 & 1
\end{array}\right|_{\varepsilon}^{2}+w_{1}\left|\begin{array}{ccc}
1 & 1 & 1 \\
1 & 1 & 1
\end{array}\right|_{\varepsilon}^{2}
$$

which gives $\varepsilon^{-1}$ as well.

Finally, suppose that $i=0$ and $i^{\prime}=1$. Notice that $(0, j, j)$ and $(1, j, j)$ are both admissible only if $j=1$. For the same reason $l=1$, and the left-hand side of $(*)$ is

$$
\left|\begin{array}{lll}
0 & 1 & 1 \\
0 & 1 & 1
\end{array}\right|_{\varepsilon} \cdot\left|\begin{array}{lll}
1 & 1 & 1 \\
0 & 1 & 1
\end{array}\right|_{\varepsilon}+w_{1}\left|\begin{array}{lll}
0 & 1 & 1 \\
1 & 1 & 1
\end{array}\right|_{\varepsilon} \cdot\left|\begin{array}{lll}
1 & 1 & 1 \\
1 & 1 & 1
\end{array}\right|_{\varepsilon},
$$

which is indeed equal to zero.

Let us denote by $\mathcal{I}_{*}^{\varepsilon}$ the colored Turaev-Viro invariants of links obtained with respect to the datum just described. 
A refinement of the formula for link-connected sums Theorem 4.9 yields the following result, that will lead to important applications in Section 7.

Theorem 5.2. Let $(M, L)$ be the link-connected sum of $\left(M_{1}, L_{1}\right)$ and $\left(M_{2}, L_{2}\right)$ along knots $K_{1} \subset L_{1}$ and $K_{2} \subset L_{2}$. Let $L_{i}^{\prime}$ be $L_{i} \backslash K_{i}$. Then

$$
\begin{aligned}
\mathcal{I}_{0}^{\varepsilon}(M, L)= & \mathcal{I}_{0}^{\varepsilon}\left(M_{1}, L_{1}\right) \cdot \mathcal{I}_{0}^{\varepsilon}\left(M_{2}, L_{2}\right) \\
+\varepsilon^{-2} & \cdot\left((\varepsilon+2) \mathcal{I}_{0}^{\varepsilon}\left(M_{1}, L_{1}^{\prime}\right)-\mathcal{I}_{0}^{\varepsilon}\left(M_{1}, L_{1}\right)\right) \\
& \quad \times\left((\varepsilon+2) \mathcal{I}_{0}^{\varepsilon}\left(M_{2}, L_{2}^{\prime}\right)-\mathcal{I}_{0}^{\varepsilon}\left(M_{2}, L_{2}\right)\right)
\end{aligned}
$$

Proof. By Theorem 4.9 we have

$\mathcal{I}_{0}^{\varepsilon}(M, L)=\mathcal{I}_{0}^{\varepsilon}\left(M_{1}, L_{1}\right) \cdot \mathcal{I}_{0}^{\varepsilon}\left(M_{2}, L_{2}\right)+\varepsilon^{-2} \mathcal{I}_{\left(0 ; K_{1}, 1\right)}^{\varepsilon}\left(M_{1}, L_{1}\right) \cdot \mathcal{I}_{\left(0 ; K_{2}, 1\right)}^{\varepsilon}\left(M_{2}, L_{2}\right)$.

Moreover, by the definition of $\mathcal{I}_{*}^{\varepsilon}$ we obtain

$$
\mathcal{I}_{\left(0 ; K_{i}, 0\right)}^{\varepsilon}\left(M_{i}, L_{i}\right)+\mathcal{I}_{\left(0 ; K_{i}, 1\right)}^{\varepsilon}\left(M_{i}, L_{i}\right)=w \mathcal{I}_{0}^{\varepsilon}\left(M_{i}, L_{i}^{\prime}\right)=(\varepsilon+2) \mathcal{I}_{0}^{\varepsilon}\left(M_{i}, L_{i}^{\prime}\right),
$$

whence the conclusion.

The $\varepsilon$-invariant of unlinks Theorem 4.8 gives the following:

Corollary 5.3. Let $U_{n}$ be the unlink with $n \geqslant 0$ components in $\mathbb{S}^{3}$. Then

$$
\mathcal{I}_{0}^{\varepsilon}\left(U_{n}\right)=(\varepsilon+2)^{n-1} .
$$

Proof. By definition $\mathcal{I}_{0}^{\varepsilon}(\emptyset)=t\left(\mathbb{S}^{3}\right)=(\varepsilon+2)^{-1}$. Furthermore, it has already been mentioned that Bing's house with the region $c$ marked (see Fig. 3 . right) is an o-spine of the unknot. If this region is colored by 0 , the only admissible coloring is when the other two regions are also colored by 0 . Hence $\mathcal{I}_{0}^{\varepsilon}\left(U_{1}\right)=1$. The desired conclusion now follows from Theorem 4.8,

Corollary 5.4. Let $\xi$ be a coloring of $U_{n}$ with at least one color 1 . Then

$$
\mathcal{I}_{\xi}^{\varepsilon}\left(U_{n}\right)=0
$$

Proof. Using the abalone, it is easy to calculate directly that $\mathcal{I}_{1}^{\varepsilon}\left(U_{1}\right)=0$. The conclusion now follows from Theorem 4.8 . 
$\mathcal{I}_{0}^{\varepsilon}$ is the $\varepsilon$-invariant of the complement Corollaries 5.3 and 5.4 suggest that the invariant $\mathcal{I}_{0}^{\varepsilon}$ is well-normalized and so is perhaps the most natural one to consider. On the other hand, as we prove in this paragraph, it has the limitation of being determined by the link complement.

Lemma 5.5. For any admissible triples $(i, j, k)$ and $(i, j, m)$ we have

$$
\left|\begin{array}{lll}
0 & i & i \\
k & j & j
\end{array}\right|_{\varepsilon} \cdot\left|\begin{array}{ccc}
0 & i & i \\
m & j & j
\end{array}\right|_{\varepsilon}=\left(w_{i} w_{j}\right)^{-1}
$$

Proof. The proof is by inspection of the possibilities.

Lemma 5.6. Let $P$ be an o-spine, and let $c$ be a length-2 region of $P$ with embedded closure. Suppose that for either edge e of $c$ the two germs of region incident to $e$ and not contained in $c$ are contained in two distinct regions. Suppose also that $c$ has a common edge with at least three distinct regions. Let $P^{\prime}$ be the marked polyhedron obtained from $P$ by removing $c$, and let $\xi^{\prime}$ be a coloring of the marked regions of $P^{\prime}$. Then there is a bijection $\imath$ : $\operatorname{Adm}_{\xi^{\prime},(c, 0)}(P) \rightarrow \operatorname{Adm}_{\xi^{\prime}}\left(P^{\prime}\right)$ such that for any $\eta \in \operatorname{Adm}_{\xi^{\prime},(c, 0)}(P)$ we have

$$
\omega(\eta)=\omega(\imath(\eta))
$$

Proof. The assumptions of the lemma imply that $P^{\prime}$ is a special polyhedron. We observe that each region of $P^{\prime}$ either corresponds to a unique region of $P$ or to the union of several (at most three) regions of $P$. Notice that in the latter case for each region $c_{1}$ in the union there is a region $c_{2}$ also in the union such the triple $c_{1}, c_{2}, c$ has a common edge. Let now $\eta \in \operatorname{Adm}_{\xi^{\prime},(c, 0)}(P)$. Since $(0,0,1)$ is not admissible, if two regions of $P$ are contained in one region of $P^{\prime}$ then they have the same color. So $\eta$ defines an admissible coloring $\imath(\eta)$ of $P^{\prime}$, and it is easy to see that this correspondence is bijective.

Since $c$ has length 2, its admissibly colored neighbourhood has the form shown in Fig. 18. Notice that, although some of the germs in Fig. 18 may belong to the same region, the total number of regions in $P^{\prime}$ is smaller than that of $P$ by 3 . Hence we see that

$$
\omega(\imath(\eta))=w_{j}^{-1} w_{k}^{-1}\left(\left|\begin{array}{ccc}
0 & j & j \\
m & k & k
\end{array}\right|_{\varepsilon} \cdot\left|\begin{array}{lll}
0 & j & j \\
n & k & k
\end{array}\right|_{\varepsilon}\right)^{-1} \omega(\eta),
$$

and, as before, we need to ensure that the factor is always equal to 1 . Since the triples $(j, k, m)$ and $(j, k, n)$ must be admissible, the conclusion follows from Lemma 5.5. 


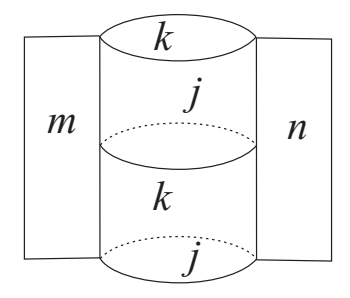

Figure 18: A colored neighbourhood of a length-2 region with embedded closure

Lemma 5.7. Every pair $(M, L)$ admits an o-spine $P$ such that each marked region of $P$ satisfies the conditions of Lemma 5.6.

Proof. Let us choose an arbitrary o-spine $P^{\prime}$ of $M$ and a projection $\Pi$ of $L$ on $P$. Of course we can and will assume that the projection has only generic intersections with itself and with $S\left(P^{\prime}\right)$ and that it decomposes each region of $P^{\prime}$ into discs. We then add a curl (as in the first Reidemeister move) on each component of $\Pi$ that has no self-intersection. We then proceed exactly as in the construction of $P_{\Pi}(L)$ for links in $\mathbb{S}^{3}$, digging a tunnel inside $P$ along $\Pi$, adding marked length-2 regions but omitting the last step (that of Fig. 8). It is easy to check that the resulting polyhedron $P$ is an o-spine of $(M, L)$ satisfying all the desired conditions.

We now recall that the $\varepsilon$-invariant [9] of a 3-manifold $M$ arising from the initial datum $\mathcal{D}_{\varepsilon}$ is denoted by $t(M)$.

Theorem 5.8. Let $L \subset M$ be a link in a closed 3-manifold $M$, and let $N_{M}(L)$ denote its complement. Then

$$
\mathcal{I}_{0}^{\varepsilon}(M, L)=t\left(N_{M}(L)\right)
$$

Proof. Let $P$ be an o-spine of $(M, L)$ as in Lemma 5.7, let $m$ be the number of empty balls in the complement $M \backslash P$, and let $P^{\prime}$ be the polyhedron obtained from $P$ by removing all the marked components. Then $P^{\prime}$ is a special skeleton (see Remark 1.5) of $N_{M}(L)$, with $N_{M}(L) \backslash P^{\prime}$ containing a collar of the boundary and $m$ balls. By repeated application of Lemma 5.6 there exists a bijection $\imath: \operatorname{Adm}_{0}(P) \rightarrow \operatorname{Adm}\left(P^{\prime}\right)$ such that for any $\eta \in \operatorname{Adm}_{0}(P)$ we have $\omega(\eta)=\omega(\imath(\eta))$. By definition, $\mathcal{I}_{0}^{\varepsilon}(L)=w^{-m} \sum_{\eta \in \operatorname{Adm}_{0}(P)} \omega(\eta)$ and

$$
t\left(N_{M}(L)\right)=w^{-m} \sum_{\eta^{\prime} \in \operatorname{Adm}\left(P^{\prime}\right)} \omega\left(\eta^{\prime}\right)=w^{-m} \sum_{\left.\eta \in \operatorname{Adm}_{(} P\right)} \omega(\imath(\eta))
$$



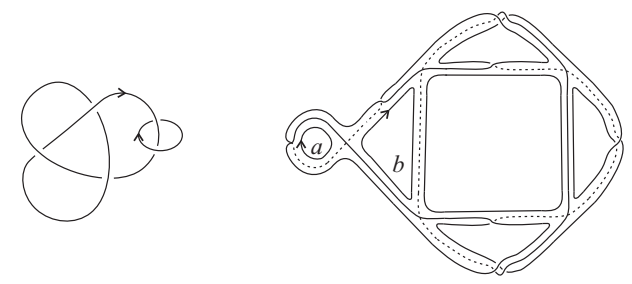

Figure 19: A low-crossing link $L$ and an o-spine of $L$. Note that the trefoil component of $L$ is dual to the region $a$ of $P$ and the unknot component of $L$ is dual to the region $b$

whence the conclusion.

In Section 8 we will show that the invariant $\mathcal{I}_{1}^{\varepsilon}$ does not have the drawback of being determined by the link complement.

Examples It follows from Corollary 5.3 that for the unknot $U_{1}$ we have

$$
\mathcal{I}_{0}^{\varepsilon}\left(U_{1}\right)=1
$$

Using the abalone (shown in Fig. 3-left) with the region $b$ marked to calculate the invariant of the trefoil $3_{1}$, we easily see that

$$
\mathcal{I}_{0}^{\varepsilon}\left(3_{1}\right)=2 .
$$

We also compute $\mathcal{I}_{0}^{\varepsilon}$ for the link $L$ shown in Fig. 19-left. An o-spine of $L$ is shown in Fig. 19-right, and a direct calculation yields

$$
\mathcal{I}_{0}^{\varepsilon}(L)=5-\varepsilon
$$

\section{Turaev-Viro invariants}

We will now discuss the Turaev-Viro initial datum introduced in [19, Section 7.1].

Definition First of all, we fix an integer $r \geqslant 3$ (called the order of the datum and of the corresponding invariant) and a root of unity $q$ of degree $2 r$ such that $q^{2}$ is a primitive root of unity of degree $r$. Recall that the quantum factorial $[n]$ ! of an integer $n \geqslant 1$ is defined by the following equalities:

$$
[n]=\frac{q^{n}-q^{-n}}{q-q^{-1}}, \quad[n] !=[n][n-1] \ldots[1] .
$$


Also by definition $[0] !=[0]=1$.

Let $\mathbb{K}$ be $\mathbb{C}$ and let $I$ be the set $\{0,1 / 2,1, \ldots,(r-3) / 2,(r-2) / 2\}$. A triple $(i, j, k)$ is admissible if it satisfies the triangle inequalities (11) and the following two conditions:

$$
i+j+k \in \mathbb{N} \text {, and } i+j+k \leqslant r-2 \text {. }
$$

The color weights and the constant $w$ are given by

$$
w_{i}=(-1)^{2 i}[2 i+1], \quad w=-\frac{2 r}{\left(q-q^{-1}\right)^{2}} .
$$

To define the $6 j$-symbols, which are called quantum $6 j$-symbols, we need the following additional functions. For every admissible triple $(i, j, k)$ we set

$$
\Delta(i, j, k)=\left(\frac{[i+j-k] ![j+k-i] ![k+i-j] !}{[i+j+k+1] !}\right)^{1 / 2}
$$

where by the square root of a real number $a$ we mean $a^{1 / 2} \geqslant 0$ if $a \geqslant 0$ and $\sqrt{-1}|a|^{1 / 2}$ if $a<0$. For every admissible 6 -tuple $(i, j, k, l, m, n)$ we also set

$$
\begin{aligned}
U(z,(i, j, k, l, m, n))=[z-i-j-k] ![z-i-m-n] ! \\
\times[z-j-l-n] ![z-k-l-m] ! \\
\begin{aligned}
L(z,(i, j, k, l, m, n))=[i+j+ & l+m-z] ! \\
\times & \times[j+k+m+n-z] ![k+i+n+l-z] !
\end{aligned}
\end{aligned}
$$

for any $z$ such that all the factorials are defined. Now we define:

$$
\begin{aligned}
\left|\begin{array}{ccc}
i & j & k \\
l & m & n
\end{array}\right|=(\sqrt{-1})^{-2(i+j+k+l+m+n)} \Delta(i, j, k) \Delta(i, m, n) \Delta(j, l, n) \Delta(k, l, m) \\
\quad \times \sum_{z}(-1)^{z}[z+1] ! U(z,(i, j, k, l, m, n))^{-1} L(z,(i, j, k, l, m, n))^{-1},
\end{aligned}
$$

where the sum is taken over all $z$ such that

$$
\begin{aligned}
\max \{i+j+k, i+ & m+n, j+l+n, k+l+m\} \leqslant z, \\
z & \leqslant \min \{i+j+l+m, j+k+m+n, k+i+n+l\} .
\end{aligned}
$$

We notice that the first condition of strong irreducibility and Conditions $(*),(* *),(* * *)$ were established in the proof of [19, Theorem 7.2]. 
Lemma 6.1. The initial datum just described is good.

Proof. To check the second condition of strong irreducibility, we need to show that, given admissible triples $(i, j, k),(i, m, n)$, there exists $l$ such that $(j, m, l)$ and $(k, n, l)$ are also admissible. Admissibility of $(j, m, l)$ means:

- $|m-j| \leqslant l \leqslant \min \{r-2-(m+j), m+j\}$;

- $l \equiv m+j(\bmod 1)$.

Similarly, admissibility of $(k, n, l)$ means

- $|n-k| \leqslant l \leqslant \min \{r-2-(k+n), k+n\} ;$

- $l \equiv k+n(\bmod 1)$.

Without loss of generality, we may and will assume that $m \geqslant j$ and $m-j \geqslant$ $|n-k|$. We remark that $m+j \equiv k+n(\bmod 1)$, and that $m-j \leqslant \min \{r-$ $2-(m+j), m+j\}$, because $m \leqslant(r-2) / 2$, being a member of an admissible triple. Thus, for the desired $l$ to exist it is sufficient to show that

- $m-j \leqslant \min \{r-2-(k+n), k+n\}$.

Using admissibility of $(i, j, k)$ and $(i, m, n)$, we see that

$$
\begin{gathered}
m-j \leqslant i+n-j \leqslant j+k+n-j=n+k, \\
m-j+n+k \leqslant m-j+n+i+j=i+m+n \leqslant r-2 .
\end{gathered}
$$

An identical argument shows that there exists $l^{\prime}$ such that $\left(k, m, l^{\prime}\right)$ and $\left(j, n, l^{\prime}\right)$ are admissible, whence the conclusion.

Let us now check the third condition. Notice that admissibility of the 6 -tuples $\left(i, j, k, j_{1}, k_{1}, i_{1}\right)$ and $\left(i, j, k, j_{2}, k_{2}, i_{2}\right)$ is equivalent to admissibility of the triples $(i, j, k),\left(i, i_{1}, k_{1}\right),\left(i, i_{2}, k_{2}\right),\left(j, i_{1}, j_{1}\right),\left(j, i_{2}, j_{2}\right),\left(k, j_{1}, k_{1}\right),\left(k, j_{2}, k_{2}\right)$. Therefore

$i_{1}+i_{2} \equiv 2 i+k_{1}+k_{2} \equiv k_{1}+k_{2} \quad(\bmod 1), \quad j_{1}+j_{2} \equiv 2 j+i_{1}+i_{2} \equiv i_{1}+i_{2} \quad(\bmod 1)$, so for the necessary $l$ to exist it is sufficient to show that

$$
\begin{aligned}
& \max \left\{\left|i_{1}-i_{2}\right|,\left|j_{1}-j_{2}\right|,\left|k_{1}-k_{2}\right|\right\} \\
\leqslant & \min \left\{i_{1}+i_{2}, j_{1}+j_{2}, k_{1}+k_{2}, r-2-\left(i_{1}+i_{2}\right), r-2-\left(j_{1}+j_{2}\right), r-2-\left(k_{1}+k_{2}\right)\right\} .
\end{aligned}
$$


Indeed, without loss of generality we will assume that $\max \left\{\left|i_{1}-i_{2}\right|, \mid j_{1}-\right.$ $\left.j_{2}|,| k_{1}-k_{2} \mid\right\}=k_{1}-k_{2}$. Then we have

$$
\begin{aligned}
& k_{1}-k_{2} \leqslant i+i_{1}-k_{2} \leqslant i_{2}+k_{2}+i_{1}-k_{2}=i_{1}+i_{2}, \\
& k_{1}-k_{2} \leqslant k+j_{1}-k_{2} \leqslant j_{2}+k_{2}+j_{1}-k_{2}=j_{1}+j_{2},
\end{aligned}
$$

and of course $k_{1}-k_{2} \leqslant k_{1}+k_{2}$. Furthermore, we have

$$
\begin{gathered}
k_{1}-k_{2}+i_{1}+i_{2} \leqslant k_{1}-k_{2}+i_{1}+i+k_{2}=k_{1}+i_{1}+i \leqslant r-2, \\
k_{1}-k_{2}+j_{1}+j_{2} \leqslant k_{1}-k_{2}+j_{1}+k+k_{2}=k_{1}+j_{1}+k \leqslant r-2, \\
k_{1}-k_{2}+k_{1}+k_{2}=2 k_{1} \leqslant r-2,
\end{gathered}
$$

whence the conclusion.

To conclude, we need to check Condition $(* * * *)$. Since by [19, Equation (19)] we have

$$
\frac{1}{w_{i} w_{j}}=\left|\begin{array}{ccc}
0 & i & i \\
k & j & j
\end{array}\right|^{2}
$$

rewriting the desired equality as $\sum_{k} \frac{w_{k}}{w_{i} w_{j}}=1$, we obtain

$$
\sum_{k} \frac{w_{k}}{w_{i} w_{j}}=\sum_{k} w_{k}\left|\begin{array}{ccc}
0 & i & i \\
k & j & j
\end{array}\right|^{2}=w_{0}^{-1}=1,
$$

where the latter two equalities are respectively by Condition $(*)$ and by direct calculation.

We denote the colored Turaev-Viro invariants corresponding to a given choice of $r, q$ by $\mathcal{I}_{*}^{r, q}$.

The invariants of unlinks The following is analogous to Corollary 5.3:

Corollary 6.2. For any $n \geqslant 0$ we have

$$
\mathcal{I}_{0}^{r, q}\left(U_{n}\right)=\left(-\frac{2 r}{\left(q-q^{-1}\right)^{2}}\right)^{n-1} .
$$


Proof. By definition $\mathcal{I}_{0}^{r, q}(\emptyset)=T V\left(\mathbb{S}^{3}\right)=\left(-\frac{2 r}{\left(q-q^{-1}\right)^{2}}\right)^{-1}$. Let us show that $\mathcal{I}_{0}^{r, q}\left(U_{1}\right)=1$ for any $r$. Indeed, we may use Bing's house with the region $c$ marked as an o-spine of the trivial knot. Whatever $r$, the only admissible coloring with color 0 on the region $c$ has color 0 on both of the other regions too. The weight of this coloring is equal to 1 whatever $r$ and $q$ are. The conclusion now follows by repeated application of Theorem 4.8 .

The analogue of Corollary 5.4 does not hold for general $r$. More precisely, we have the following:

Proposition 6.3. There exist infinitely many pairs $r, q$ such that $\mathcal{I}_{2}^{r, q}$ of the unknot is not equal to 0 .

Proof. We use the abalone with the region $a$ marked to calculate the invariants of the unknot. By definition we have

$$
\mathcal{I}_{2}^{r, q}\left(U_{1}\right)=w_{0} w_{2}\left|\begin{array}{lll}
0 & 2 & 2 \\
2 & 2 & 2
\end{array}\right|+w_{2}^{2}\left|\begin{array}{lll}
2 & 2 & 2 \\
2 & 2 & 2
\end{array}\right|+w_{2} w_{4}\left|\begin{array}{lll}
4 & 2 & 2 \\
2 & 2 & 2
\end{array}\right| \text { for } r \geqslant 6 .
$$

By direct calculation for all $r \geqslant 6$ we have

$$
\mathcal{I}_{2}^{r, q}\left(U_{1}\right)=1+[3]^{2} \frac{[5]-1}{[4] !}-\frac{1}{[4]}
$$

and it is sufficient to show that for infinitely many $r$ 's and some choice of $q$ we have

$$
[2][4]+[3][5]-[3]-[2] \neq 0 \text {. }
$$

Let us take $q=e^{(\pi \cdot \sqrt{-1}) / r}$. By definition we have

$$
\begin{aligned}
& {[2][4]+[3][5]-[3]-[2]=} \\
& =\frac{q^{8}+q^{-8}+q^{6}+q^{-6}-\left(q^{4}+q^{-4}\right)-\left(q^{2}+q^{-2}\right)-\left(q^{3}+q^{-3}\right)+q+q^{-1}}{\left(q-q^{-1}\right)^{2}} .
\end{aligned}
$$

For the chosen $q$ this is equal to 0 if and only if:

$$
\cos \frac{8 \pi}{r}+\cos \frac{6 \pi}{r}-\cos \frac{4 \pi}{r}-\cos \frac{3 \pi}{r}-\cos \frac{2 \pi}{r}+\cos \frac{\pi}{r}=0 .
$$

If this holds, $\cos \frac{\pi}{r}$ must be a solution of a specific equation of degree 8 with integer coefficients, namely the equation $128 t^{8}-224 t^{6}+104 t^{4}-4 t^{3}-8 t^{2}+4 t=$ 0 . Since this equation has at most 8 real roots, we get the conclusion. 
$\mathcal{I}_{0}^{r, q}$ and the $T V$ invariant of the complement Here we show that, just as in the case of $\mathcal{I}_{0}^{\varepsilon}$, the invariants $\mathcal{I}_{0}^{r, q}$ are determined by the link complements. Namely, we obtain the following:

Theorem 6.4. Let $L \subset M$ be a link in a closed 3-manifold $M$, and let $N_{M}(L)$ be its complement. Then

$$
\mathcal{I}_{0}^{r, q}(L)=T V\left(N_{M}(L)\right)
$$

The proof imitates that of Theorem 5.8, using Lemma 5.7 and the analogue of Lemma 5.6, which in turn is established using [19, Equation (19)] in place of Lemma 5.5.

Relations with the $\varepsilon$-invariant It is well-known that the 3 -manifold $\varepsilon$ invariant coincides with the homologically trivial part of the Turaev-Viro invariant of order 5 for a suitable choice of $q$. It turns out that in the case of knots in the sphere an even stronger statement holds.

Theorem 6.5. Let $K$ be a knot in $\mathbb{S}^{3}$. Then

$$
\mathcal{I}_{0}^{\varepsilon}(K)=\mathcal{I}_{0}^{5, q}(K)
$$

for any choice of $\varepsilon$ and a suitable choice of $q$.

Lemma 6.6. Let $K \subset \mathbb{S}^{3}$ be a knot. Then for any $r$ and for any projection $\Pi$ of $K$ any coloring in $\operatorname{Adm}_{0}\left(P_{\Pi}(K)\right)$ involves integer colors only.

Proof. An admissible coloring on $P_{\Pi}(K)$ can be re-interpreted in the following way. We assign a color to each region of the diagram $\Pi$, to each overpass, to each underpass, to each crossing (this is the color of the length- 4 region arising from the tunnel going over itself, see Fig. (6), and we choose two more numbers, corresponding to the colors of the length-1 regions arising with the "puncturing" move (see Fig. 8). Notice that the underpass and the overpass at the point where we add the transverse disc must have the same color, and one of the regions adjacent to them must have the same color as well. It follows that this color is an integer. The region on the other side must also have an integer color by admissibility.

It is easy to observe that any two regions separated by one edge of the diagram must have colors that are either both integers or both half-integers. Thus, since one region has integer color, the same should be true for all 
other regions. Hence if an overpass and an underpass intersect, their colors are either both integers or both half-integers. Therefore the colors of all overpasses and of all underpasses are either all integers or all half-integers; by the above, they must be all integers.

It remains to check the conclusion for the regions corresponding to crossing points and for the length-1 discs of Fig. 8. But all these regions have embedded closures, no two of them are adjacent to the same edge and all the other regions have already been shown to have integer colors. Then by admissibility all these regions must indeed have integer colors.

Proof of Theorem 6.5. Notice that any admissible coloring in the sense of the initial datum $\mathcal{D}_{\varepsilon}$ is also an admissible coloring in the sense of any TuraevViro initial datum of order 5. If we choose $q$ to be such that $\varepsilon=q+q^{-1}$, which we can always do, this correspondence preserves the weights of the colors and the values of quantum $6 j$-symbols, whence the conclusion.

We remark that calculation of $\mathcal{I}_{0}^{\varepsilon}$ is much easier than the calculation of

$\mathcal{I}_{0}^{5, q}$, hence for knots it is more effective to study $\mathcal{I}_{0}^{\varepsilon}$ rather than $\mathcal{I}_{0}^{5, q}$. On the other hand, there exist links for which Theorem 6.5 does not hold.

\section{Relations with polynomial invariants}

In this section we consider the relation between the colored Turaev-Viro invariants and some of the well-known polynomial invariants of links, namely, the HOMFLY polynomial, the Kauffman polynomial, and the Alexander polynomial.

Theorem 7.1. There exists a pair of links in $\mathbb{S}^{3}$ such that their HOMFLY polynomials are the same and their Kauffman polynomials are the same but their $\varepsilon$-invariants $\mathcal{I}_{0}^{\varepsilon}$ are distinct.

Proof. Recall that both the Kauffman polynomial $F_{L}(a, z)$ and the HOMFLY polynomial $P_{L}(a, z)$ are multiplicative under connected sum of links. In particular, let $L=K_{1} \sqcup K_{2}$ be a two-component link, and let $K$ be a knot. Denote by $L^{(i)}$ the link obtained by taking the connected sum of $L$ with $K$ along $K_{i}$. Then:

$$
\begin{aligned}
& F_{L^{(1)}}(a, z)=F_{L}(a, z) \cdot F_{K}(a, z)=F_{L^{(2)}}(a, z), \\
& P_{L^{(1)}}(a, z)=P_{L}(a, z) \cdot P_{K}(a, z)=P_{L^{(2)}}(a, z) .
\end{aligned}
$$



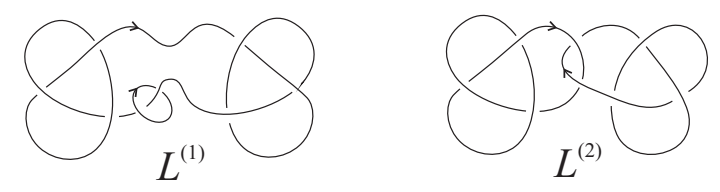

Figure 20: The links $L^{(1)}$ and $L^{(2)}$
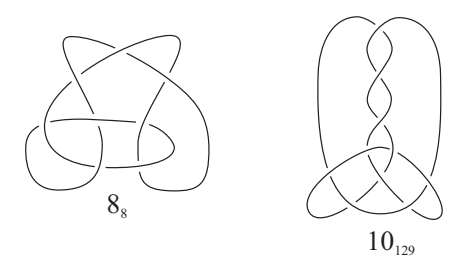

Figure 21: Prime knots with the same HOMFLY polynomial and distinct Turaev-Viro invariants

Let us give an example of $L$ and $K$ such that $\mathcal{I}_{0}^{\varepsilon}\left(L^{(1)}\right) \neq \mathcal{I}_{0}^{\varepsilon}\left(L^{(2)}\right)$. Take $K$ to be a trefoil and take $L$ to be the link shown in Fig. 19. Denote by $K_{1}$ the trefoil component of $L$ and by $K_{2}$ the trivial component of $L$. The links $L^{(1)}$ and $L^{(2)}$ are shown in Fig. 20, Then by Theorem 5.2 and Corollary 5.3 we have:

$$
\begin{aligned}
\mathcal{I}_{0}^{\varepsilon}\left(L^{(1)}\right) & =\mathcal{I}_{0}^{\varepsilon}(L) \cdot \mathcal{I}_{0}^{\varepsilon}\left(3_{1}\right) \\
+ & \varepsilon^{-2}\left((\varepsilon+2) \mathcal{I}_{0}^{\varepsilon}\left(U_{1}\right)-\mathcal{I}_{0}^{\varepsilon}(L)\right) \cdot\left((\varepsilon+2) \mathcal{I}_{0}^{\varepsilon}(\emptyset)-\mathcal{I}_{0}^{\varepsilon}\left(3_{1}\right)\right)=18-7 \varepsilon
\end{aligned}
$$

At the same time we have

$$
\begin{aligned}
\mathcal{I}_{0}^{\varepsilon}\left(L^{(2)}\right) & =\mathcal{I}_{0}^{\varepsilon}(L) \cdot \mathcal{I}_{0}^{\varepsilon}\left(3_{1}\right) \\
& +\varepsilon^{-2}\left((\varepsilon+2) \mathcal{I}_{0}^{\varepsilon}\left(3_{1}\right)-\mathcal{I}_{0}^{\varepsilon}(L)\right) \cdot\left((\varepsilon+2) \mathcal{I}_{0}^{\varepsilon}(\emptyset)-\mathcal{I}_{0}^{\varepsilon}\left(3_{1}\right)\right)=15-6 \varepsilon
\end{aligned}
$$

whence the conclusion.

Remark 7.2. The links constructed in the proof of the above theorem are reducible, but there also exists pairs of prime knots with the same HOMFLY polynomial but distinct Turaev-Viro invariants of order 7. One such example is given by the knots $8_{8}$ and $10_{129}$ in the tables of Tait [14] and Little [8] (see Fig. 21), which have the same HOMFLY polynomial [6]. We have calculated 
their invariants $\mathcal{I}_{0}^{7, q}$ and $\mathcal{I}_{0}^{8, q}$ using Theorem 5.8 and the program "Threemanifold Recognizer" [11], which, among other things, calculates TuraevViro invariants of link complements in $\mathbb{S}^{3}$. The results were:

$$
\begin{aligned}
& \mathcal{I}_{0}^{7, q}\left(8_{8}\right)=71\left(q^{2}+q^{-2}\right)-181\left(q+q^{-1}\right)+240 \\
& \mathcal{I}_{0}^{7, q}\left(10_{129}\right)=89\left(q^{2}+q^{-2}\right)-227\left(q+q^{-1}\right)+301 \\
& \mathcal{I}_{0}^{8, q}\left(8_{8}\right)=79-54\left(q^{2}+q^{-2}\right), \\
& \mathcal{I}_{0}^{8, q}\left(1_{129}\right)=31-20\left(q^{2}+q^{-2}\right) .
\end{aligned}
$$

Proposition 7.3. There exists a pair of knots with the same Alexander polynomial but distinct $\varepsilon$-invariants.

Proof. Let $W(K)$ denote the Whitehead double of a given knot $K \subset \mathbb{S}^{3}$. Then it is known that the Alexander polynomial of $W(K)$ is equal to 1 . On the other hand, a direct (but computer-aided) calculation allowed us to obtain that

$$
\mathcal{I}_{0}^{\varepsilon}\left(W\left(3_{1}\right)\right)=5-2 \varepsilon, \quad \mathcal{I}_{0}^{\varepsilon}\left(W\left(4_{1}\right)\right)=4-\varepsilon
$$

\section{Colored Turaev-Viro invariants not determined by link complements}

Theorems 5.8 and 6.4 show that some colored Turaev-Viro invariants of a link are determined by the link complement. We now show that not all of them are.

Proposition 8.1. There exists a pair of links in $\mathbb{S}^{3}$ with homeomorphic complements but distinct $\mathcal{I}_{1}^{\varepsilon}$ invariants.

Proof. Consider the links obtained by closing the braids $\sigma_{2} \sigma_{1}^{2} \sigma_{2}$ and $\sigma_{1}^{2} \sigma_{2} \sigma_{1}^{2} \sigma_{2}$, see Fig. 22. Since they are obtained from each other by a full twist around a disc spanning the common unknotted component, they have the same complements. To compute their $\mathcal{I}_{1}^{\varepsilon}$, we constructed o-spines from the projections, obtaining the polyhedra of Fig. 23. A direct computation then shows that

$$
\begin{gathered}
\mathcal{I}_{1}^{\varepsilon}\left(\widehat{\sigma_{2} \sigma_{1}^{2} \sigma_{2}}\right)=-(\varepsilon+2), \\
\mathcal{I}_{1}^{\varepsilon}\left(\widehat{\sigma_{1}^{2} \sigma_{2} \sigma_{1}^{2}} \sigma_{2}\right)=-(4 \varepsilon+3) .
\end{gathered}
$$




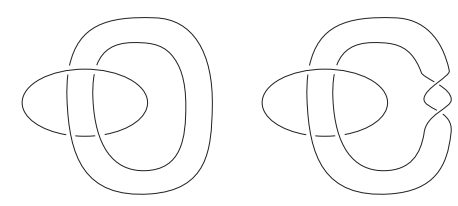

Figure 22: Two links with the same complement
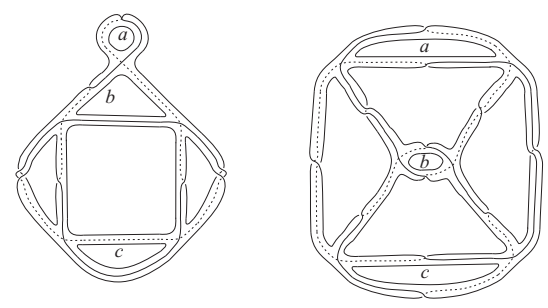

Figure 23: O-spines of the links of Fig. 22. The letters $a, b, c$ denote the regions dual to the link components

\section{References}

[1] G. Amendola, A calculus for ideal triangulations of three-manifolds with embedded arcs, Math. Nachr. (9) 278 (2005), 975-994.

[2] R. Askey - J. WiLson, A set of orthogonal polynomials that generalize the Racah coefficients or $6-j$ symbols, SIAM J. Math. Anal. (5) 10 (1979), 1008-1016.

[3] J. Barrett - J. García-Islas - J. Martins, Observables in the Turaev-Viro and Crane-Yetter models, J. Math. Phys. (9) 48 (2007), 18 pp.

[4] R. Benedetti - C. Petronio, "Branched Standard Spines of 3Manifolds," Lecture Notes in Math., Vol. 1653, Springer-Verlag, Berlin, 1997.

[5] B. G. CASLER, An embedding theorem for connected 3-manifolds with boundary, Proc. Amer. Math. Soc. 16 (1965), 559-566.

[6] V. Jones, Hecke algebra representations of braid groups and link polynomials, Ann. of Math. 126 (1987), 335-388. 
[7] A. Kirillov - N. Reshetikhin, Representations of the algebra $U_{q}\left(s_{2}\right)$, q-orthogonal polynomials and invariants of links, In: V.G. Kac (ed.), "Infinite-dimensional Lie Algebras and Groups," Adv. Ser. In Math. Phys., Vol. 7, World Scientific, Singapore, 1988, pp. 285-339.

[8] C.N. Little, Non-alternate \pm knots, Trans. Roy. Soc. Edinburgh. 39 (1900), 771-778.

[9] S. Matveev - M. Sokolov, On a simple invariant of Turaev-Viro type, J. Math. Sci. (New York) (2) 94 (1999), 1226-1229.

[10] S. Matveev, Algorithmic topology and classification of 3-manifolds, Springer-Verlag, 2003.

[11] S. Matveev, Three-manifold recognizer, computer program available at www.csu.ac.ru/ $\operatorname{trk} /$ spine.

[12] C. Petronio, Complexity of 3-orbifolds, Topology Appl. 153 (2006), 1658-1681.

[13] N. Reshetikhin - V. TuRAeV, Invariants of 3-manifolds via link polynomials and quantum groups, Invent. Math. 101 (1992), 547-597.

[14] P.G. TaIt, On knots I, II, III, In: "Scientific Papers I," Cambridge University Press, London, 1898, pp. 273-437.

[15] V. Turaev, The Yang-Baxter equation and invariants of links, Invent. Math. 92 (1988), 527-553.

[16] V. Turaev, Shadow links and face models of statistical mechanics, J. Differential Geom. 36 (1992), 35-74.

[17] V. Turaev, Quantum invariants of links and 3-valent graphs in 3manifolds, Publ. Math. Inst. Hautes Études Sci. 77 (1993), 121-171.

[18] V. Turaev, "Quantum Invariants of Knots and 3-Manifolds," de Gruyter Studies in Math., Vol. 18, 1994.

[19] V. Turaev - O. Viro, State sum invariants of 3-manifolds and quantum 6j-symbols, Topology 31 (1992), 865-902. 
Dipartimento di Matematica Applicata Università di Pisa

Via Filippo Buonarroti, 1C

I-56127, PISA

Italy

pervova@guest.dma.unipi.it

petronio@dm.unipi.it 